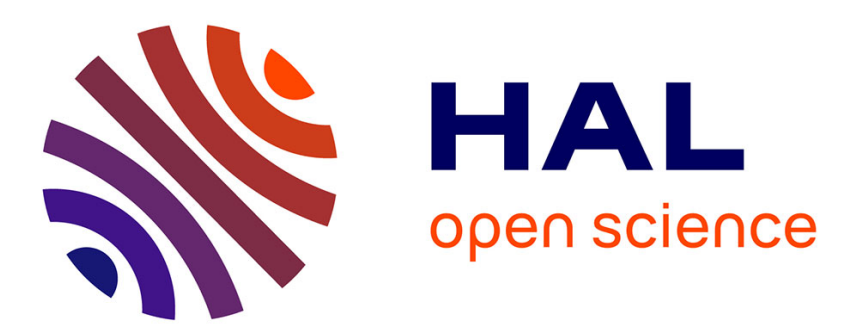

\title{
A quasi-optimal domain decomposition algorithm for the time-harmonic Maxwell's equations
}

\author{
Mohamed El Bouajaji, Bertrand Thierry, X Antoine, C Geuzaine
}

\section{To cite this version:}

Mohamed El Bouajaji, Bertrand Thierry, X Antoine, C Geuzaine. A quasi-optimal domain decomposition algorithm for the time-harmonic Maxwell's equations. Journal of Computational Physics, 2015, 294 (1), pp.38-57. 10.1016/j.jcp.2015.03.041 . hal-01095566

\section{HAL Id: hal-01095566 https://hal.science/hal-01095566}

Submitted on 9 Feb 2016

HAL is a multi-disciplinary open access archive for the deposit and dissemination of scientific research documents, whether they are published or not. The documents may come from teaching and research institutions in France or abroad, or from public or private research centers.
L'archive ouverte pluridisciplinaire HAL, est destinée au dépôt et à la diffusion de documents scientifiques de niveau recherche, publiés ou non, émanant des établissements d'enseignement et de recherche français ou étrangers, des laboratoires publics ou privés. 


\title{
A quasi-optimal domain decomposition algorithm for the time-harmonic Maxwell's equations
}

\author{
M. El Bouajaji ${ }^{1}$, B. Thierry ${ }^{2,3}$, X. Antoine ${ }^{1}$ and C. Geuzaine ${ }^{2}$
}

\begin{abstract}
This paper presents a new non-overlapping domain decomposition method for the time harmonic Maxwell's equations, whose effective convergence is quasi-optimal. These improved properties result from a combination of an appropriate choice of transmission conditions and a suitable approximation of the Magnetic-to-Electric operator. A convergence theorem of the algorithm is established and numerical results validating the new approach are presented.
\end{abstract}

Keywords: Maxwell's equation, Electromagnetism, Domain decomposition methods, Finite elements, Padé approximants.

\section{Introduction}

In terms of computational methods, solving three-dimensional time-harmonic electromagnetic wave problems is known to be a challenging topic, especially in the high frequency regime. Among the various approaches that can be used to solve such problems, the Finite Element Method (FEM) with an Absorbing Boundary Condition (ABC) or a Perfectly Matched Layer (PML) is widely used for its ability to handle complex geometrical configurations and materials with non-homogeneous electromagnetic properties [20]. However, the brute-force application of the FEM in the highfrequency regime leads to the solution of very large, complex and possibly indefinite linear systems. Direct sparse solvers do not scale well for such problems, and Krylov subspace iterative solvers can exhibit slow convergence, or even diverge [16]. Domain decomposition methods provide an alternative, iterating between subproblems of smaller sizes, amenable to sparse direct solvers.

Improving the convergence properties of the iterative process constitutes the key in designing an effective algorithm, in particular in the high frequency regime. The optimal convergence is obtained by using as transmission condition on each interface between subdomains the so-called Magnetic-to-Electric (MtE) map [27] linking the magnetic and the electric surface currents on the interface. This however leads to a very expensive procedure in practice, as the MtE operator is non-local. A great variety of techniques based on local transmission conditions have therefore been proposed to build practical algorithms [10, 1, 12, 15, 29, 28, 30, 11].

In the context of acoustic simulations, quasi-optimal local transmission boundary conditions for domain decomposition methods were proposed in [7], based on high-order rational approximations of the Dirichlet-to-Neumann operator. In this paper, we analyze and validate the extension of this approach to electromagnetics, based on the high-order approximations of the MtE operator developed in [14]. We show that the resulting domain decomposition algorithm exhibits quasioptimal convergence properties, i.e., that the convergence is optimal for the evanescent modes and significantly improved compared to competing approaches for the remaining modes. The finite

\footnotetext{
${ }^{1}$ Institut Elie Cartan de Lorraine, Université de Lorraine, CNRS UMR 7502, INRIA CORIDA team, Boulevard des Aiguillettes B.P. 239, F-54506 Vandoeuvre-lès-Nancy, France (Mohamed.El_Bouajaji@inria.fr, Xavier.Antoine@univ-lorraine.fr).

${ }^{2}$ Université de Liège, Institut Montéfiore B28, B-4000 Liège, Belgique (CGeuzaine@ulg.ac.be).

${ }^{3}$ Laboratoire J.L. Lions (LJLL), University of Paris VI, Paris, France (thierry@ljll.math.upmc.fr).
} 
element implementation of the method is available online as the open source software package GetDDM $^{1}$, which combines the open source mesh generator Gmsh [19] and the finite element solver GetDP $[13,18]$ for large scale domain decomposition simulations.

The paper is organized as follows. In Section 2 we introduce the scattering problem as well as the non-overlapping DDM. Optimized local transmission boundary conditions are presented in Section 3. Section 4 develops a convergence analysis for this approximate transmission condition on a model problem. Section 5 details the complex Padé approximation of the square-root operator to get a local representation. Section 6 presents the finite element implementation of the resulting DDM. Numerical results on three-dimensional problems are presented in Section 7. The paper is concluded in Section 8 with perspectives for future work.

\section{Problem setting and non-overlapping optimized Schwarz DDM}

Let $K$ be a bounded scatterer in $\mathbb{R}^{3}$ with smooth closed boundary $\Gamma$. The associated unbounded domain of propagation is denoted by $\Omega:=\mathbb{R}^{3} \backslash \bar{K}$. The exterior electromagnetic scattering problem by a perfectly conducting body $K$ is given by

$$
\begin{cases}\operatorname{curl} \operatorname{curl} \mathbf{E}-k^{2} \mathbf{E}=0, & \text { in } \Omega, \\ \gamma^{T}(\mathbf{E})=-\gamma^{T}\left(\mathbf{E}^{\text {inc }}\right), & \text { on } \Gamma, \\ \lim _{r \rightarrow \infty} r\left(\mathbf{E}-\frac{\imath}{k} \hat{\mathbf{x}} \times \operatorname{curl} \mathbf{E}\right)=0 .\end{cases}
$$

In the above equations, $\mathbf{E}$ denotes the scattered electric field. The wavenumber is $k:=2 \pi / \lambda$, where $\lambda$ is the wavelength, and the unit imaginary number is $\imath=\sqrt{-1}$. The curl operator is defined by curl $\mathbf{a}:=\nabla \times \mathbf{a}$, for a complex-valued vector field $\mathbf{a} \in \mathbb{C}^{3}$. The nabla operator is $\nabla:={ }^{t}\left(\partial_{x_{1}}, \partial_{x_{2}}, \partial_{x_{3}}\right)$, where $\mathbf{x}={ }^{t}\left(x_{1}, x_{2}, x_{3}\right) \in \mathbb{R}^{3}$. The notation $\mathbf{a} \times \mathbf{b}$ designates the cross product and $\mathbf{a} \cdot \overline{\mathbf{b}}$ the inner product between two vectors $\mathbf{a}$ and $\mathbf{b}$ in $\mathbb{C}^{3}$, where $\bar{z}$ is the complex conjugate of $z \in \mathbb{C}$. The associated norm is $\|\mathbf{a}\|:=\sqrt{\mathbf{a} \cdot \overline{\mathbf{a}}}$. Vector $\mathbf{n}$ is the unit outwardly directed normal to $\Omega$ and $\mathbf{E}^{\text {inc }}$ defines a given incident electric field. Let us consider a general domain $\mathcal{D}$ with boundary $\partial \mathcal{D}, \mathbf{n}$ the outwardly directed unit vector to $\mathcal{D}$, then the tangential traces applications are defined by

$$
\gamma^{t}: \mathbf{v} \mapsto \mathbf{v}_{t}:=\mathbf{n} \times \mathbf{v}_{\mid \partial \mathcal{D}} \quad \text { and } \quad \gamma^{T}: \mathbf{v} \mapsto \mathbf{v}_{T}:=\mathbf{n} \times\left(\mathbf{v}_{\mid \partial \mathcal{D}} \times \mathbf{n}\right) .
$$

Let us now write $\mathbf{x}=r \hat{\mathbf{x}} \in \mathbb{R}^{3}$, where $r:=\|\mathbf{x}\|$ is the radial distance to the origin and $\hat{\mathbf{x}}$ is the directional vector of the unit sphere $\mathbb{S}_{1}$. Then, the last equation of system (1), which is the socalled Silver-Müller radiation condition at infinity, provides the uniqueness of the solution to the scattering boundary-value problem (1).

To numerically solve (1) by a volume discretization method, it is standard to truncate the exterior domain of propagation by using a fictitious boundary $\Gamma^{\infty}$ surrounding $\Omega$. As a result, we have to solve the following problem in a bounded domain $\Omega$, with boundaries $\Gamma$ and $\Gamma_{\infty}$,

$$
\begin{cases}\text { curl curl } \mathbf{E}-k^{2} \mathbf{E}=0, & \text { in } \Omega, \\ \gamma^{T}(\mathbf{E})=-\gamma^{T}\left(\mathbf{E}^{\text {inc }}\right), & \text { on } \Gamma, \\ \mathcal{B}\left(\gamma^{T}(\mathbf{E})\right)-\frac{\imath}{k} \gamma^{t}(\operatorname{curl} \mathbf{E})=0, & \text { on } \Gamma^{\infty} .\end{cases}
$$

The operator $\mathcal{B}$ can be exact, resulting then in a transparent boundary condition that avoids any spurious unphysical reflection. However, such a boundary condition is global since it is defined by a nonlocal boundary integral operator on $\Gamma_{\infty}$ (i.e. the MtE operator $\Lambda: \gamma^{T}(\mathbf{E}) \mapsto \Lambda\left(\gamma^{T}(\mathbf{E})\right)=$ $\left.\gamma^{t}(\mathbf{c u r l} \mathbf{E})\right)$. This generates a dense part in the global discretization matrix that must be solved at the end of the computational process. For reducing the cost of computation, a local Absorbing

\footnotetext{
${ }^{1}$ http://onelab.info/wiki/GetDDM.
} 
Boundary Condition $(\mathrm{ABC})$ is generally preferred, which means that the operator $\mathcal{B}$ is in fact an approximation of $\Lambda$. Since the aim of this paper is not devoted to ABCs, we restrict ourselves to the simplest ABC: $\mathcal{B}=\mathbf{I}$ (I is the surface identity operator). This corresponds to the well-known Silver-Müller ABC at finite distance.

Let us now focus on the construction of optimized Schwarz Domain Decomposition Methods (DDM) without overlap [17, 12, 15, 23, 22, 29, 28, 7, 1, 10, 9, 30, 11] for the approximate boundaryvalue problem (2). The first step of the method $[9,10]$ consists in splitting $\Omega$ into several subdomains $\Omega_{i}, i=1, \ldots, N_{\mathrm{dom}}$, in such a way that

- $\bar{\Omega}=\bigcup_{i=1}^{N_{\text {dom }}} \bar{\Omega}_{i}\left(i=1, \ldots, N_{\text {dom }}\right)$,

- $\Omega_{i} \cap \Omega_{j}=\emptyset$, if $i \neq j,\left(i, j=1, \ldots, N_{\mathrm{dom}}\right)$,

- $\partial \Omega_{i} \cap \partial \Omega_{j}=\bar{\Sigma}_{i j}=\bar{\Sigma}_{j i}\left(i, j=1, \ldots, N_{\text {dom }}\right)$ is the fictitious interface separating $\Omega_{i}$ and $\Omega_{j}$ as long as its interior $\Sigma_{i j}$ is not empty.

In a second step, we solve smaller size problems on each subdomain $\Omega_{i}$ by an iterative process (indexed by $p$ ) and using transmission boundary conditions (defined by an operator $\mathcal{S}$ below): we compute $\mathbf{E}_{i}^{p+1}, 1 \leq i \leq N_{\text {dom }}$, from $\mathbf{E}_{j}^{p}, 1 \leq j \neq i \leq N_{\text {dom }}$, by

$$
\begin{cases}\operatorname{curl} \mathbf{c u r l} \mathbf{E}_{i}^{p+1}-k^{2} \mathbf{E}_{i}^{p+1}=\mathbf{0}, & \text { in } \Omega_{i}, \\ \gamma_{i}^{T}\left(\mathbf{E}_{i}^{p+1}\right)=-\gamma_{i}^{T}\left(\mathbf{E}_{i}^{\text {inc }}\right), & \text { on } \Gamma_{i}, \\ \gamma_{i}^{T}\left(\mathbf{E}_{i}^{p+1}\right)-\frac{\imath}{k} \gamma_{i}^{t}\left(\mathbf{c u r l} \mathbf{E}_{i}^{p+1}\right)=\mathbf{0}, & \text { on } \Gamma_{i}^{\infty}, \\ \mathcal{S}\left(\gamma_{i}^{T}\left(\mathbf{E}_{i}^{p+1}\right)\right)-\frac{\imath}{k} \gamma_{i}^{t}\left(\mathbf{c u r l} \mathbf{E}_{i}^{p+1}\right)=\mathcal{S}\left(\gamma_{j}^{T}\left(\mathbf{E}_{j}^{p}\right)\right)+\frac{\imath}{k} \gamma_{j}^{t}\left(\operatorname{curl} \mathbf{E}_{j}^{p}\right):=\mathbf{g}_{i j}^{p}, \quad \text { on } \Sigma_{i j},\end{cases}
$$

and then form the quantities $\mathbf{g}_{j i}^{p+1}$ through

$$
\mathbf{g}_{j i}^{p+1}=\mathcal{S}\left(\gamma_{i}^{T}\left(\mathbf{E}_{i}^{p+1}\right)\right)+\frac{\imath}{k} \gamma_{i}^{t}\left(\operatorname{curl} \mathbf{E}_{i}^{p+1}\right)=-\mathbf{g}_{i j}^{p}+2 \mathcal{S}\left(\gamma_{i}^{T}\left(\mathbf{E}_{i}^{p+1}\right)\right), \quad \text { on } \Sigma_{i j},
$$

where $\mathbf{E}_{i}=\mathbf{E}_{\mid \Omega_{i}}, \mathbf{n}_{i}\left(\operatorname{resp} . \mathbf{n}_{j}\right)$ is the outward unit normal to $\Omega_{i}\left(\operatorname{resp} . \Omega_{j}\right), i, j=1, \ldots, N_{\text {dom }}$, $\Gamma_{i}=\partial \Omega_{i} \cap \Gamma, \Gamma_{i}^{\infty}=\partial \Omega_{i} \cap \Gamma^{\infty}$ and $\mathcal{S}$ is an inversible transmission operator through the interfaces $\Sigma_{i j}$. Let us remark that the boundary condition on $\Gamma_{i}$ (resp. $\Gamma_{i}^{\infty}$ ) does not take place if the interior of $\partial \Omega_{i} \cap \Gamma$ (resp. $\partial \Omega_{i} \cap \Gamma^{\infty}$ ) is the empty set. The operators $\gamma_{i}^{t}$ and $\gamma_{i}^{T}$ are moreover given by

$$
\gamma_{i}^{t}: \mathbf{v}_{i} \mapsto \mathbf{v}_{i}^{t}:=\mathbf{n}_{i} \times\left.\mathbf{v}_{i}\right|_{\partial \mathcal{D}} \quad \text { and } \quad \gamma_{i}^{T}: \mathbf{v}_{i} \mapsto \mathbf{v}_{i}^{T}:=\mathbf{n}_{i} \times\left(\left.\mathbf{v}_{i}\right|_{\partial \mathcal{D}} \times \mathbf{n}_{i}\right)
$$

Solving at each step all the local transmission problems through (3)-(4) may be rewritten as one application of the iteration operator $\mathcal{A}: \quad \times_{i, j=1}^{N_{\text {dom }}}\left(L^{2}\left(\Sigma_{i j}\right)\right)^{3} \mapsto \times_{i, j=1}^{N_{\text {dom }}}\left(L^{2}\left(\Sigma_{i j}\right)\right)^{3}$ defined by

$$
\mathbf{g}^{p+1}=\mathcal{A} \mathbf{g}^{p}+\mathbf{b}
$$

where $\mathbf{g}^{p}$ is the set of boundary data $\left(\mathbf{g}_{j i}^{p}\right)_{1 \leq i, j \leq N_{d o m}}$, and $\mathbf{b}$ is given by the incident wave field boundary data. Therefore, (3)-(4) can be interpreted as an iteration step of the Jacobi fixed point iteration method applied to the linear system

$$
(\mathcal{I}-\mathcal{A}) \mathbf{g}=\mathbf{b}
$$

where $\mathcal{I}$ is the identity matrix of size $N_{\text {dom }}^{2} \times N_{\text {dom }}^{2}$. A consequence is that any Krylov subspace iterative solver could be used for solving this equation. This can significantly improve the convergence rate of the method most particularly if $\mathcal{S}$ is well-chosen. 


\section{Optimized transmission boundary conditions}

\subsection{Surface operators}

In the sequel, we make use of some surface operators for a general smooth three-dimensional surface $\Gamma$. We introduce these operators here and precise the associated functional spaces.

The tangential gradient $\nabla_{\Gamma}$ is an operator from $H^{1 / 2}(\Gamma)$ to $\mathbf{H}_{\times}^{-1 / 2}(\Gamma)$, where $\mathbf{H}_{\times}^{s}(\Gamma):=\{\mathbf{v} \in$ $\mathbf{H}^{s}(\Gamma) / \mathbf{v} \cdot \mathbf{n}=0$ on $\left.\Gamma\right\}=\mathbf{n} \times \mathbf{H}^{s}$, for $s \in \mathbb{R}$. The operator $\operatorname{curl}_{\Gamma}$ is the tangential vector curl operator which acts from $H^{1 / 2}(\Gamma)$ to $\mathbf{H}_{\times}^{-1 / 2}(\Gamma)$ (the duality is defined with respect to the $L^{2}$ and $\mathbf{L}^{2}$-inner products). These operators have dual operators $\operatorname{div}_{\Gamma}$ and $\operatorname{curl}_{\Gamma}$, respectively, which apply from $\mathbf{H}_{\times}^{1 / 2}(\Gamma)$ to $H^{-1 / 2}(\Gamma)$. The scalar Laplace-Beltrami operator is defined by $\Delta_{\Gamma}:=$ $\operatorname{div}_{\Gamma} \nabla_{\Gamma}=-\operatorname{curl}_{\Gamma} \operatorname{curl}_{\Gamma}$. The vectorial Laplace-Beltrami operator is: $\boldsymbol{\Delta}_{\Gamma}=\nabla_{\Gamma} \operatorname{div}_{\Gamma}-\operatorname{curl}_{\Gamma} \operatorname{curl}_{\Gamma}$. We introduce now $\mathbf{H}_{\times}^{-1 / 2}\left(\operatorname{div}_{\Gamma}, \Gamma\right):=\left\{\mathbf{v} \in \mathbf{H}_{\times}^{-1 / 2}(\Gamma) / \operatorname{div}_{\Gamma} \mathbf{v} \in H^{-1 / 2}(\Gamma)\right\}$ which is the Hilbert space of well-defined surface divergence fields. Its duals is: $\mathbf{H}_{\times}^{-1 / 2}\left(\operatorname{curl}_{\Gamma}, \Gamma\right):=\left\{\mathbf{v} \in \mathbf{H}_{\times}^{-1 / 2}(\Gamma) /\right.$ $\left.\operatorname{curl}_{\Gamma} \mathbf{v} \in H^{-1 / 2}(\Gamma)\right\}$. In the case of a smooth domain, the applications $\gamma^{t}$ and $\gamma^{T}$ can be extended by continuity to surjective linear applications from $\mathbf{H}(\mathbf{c u r l}, \Omega)$ to $\mathbf{H}_{\times}^{-1 / 2}\left(\operatorname{div}_{\Gamma}, \Gamma\right)$ and $\mathbf{H}_{\times}^{-1 / 2}\left(\operatorname{curl}_{\Gamma}, \Gamma\right)$, respectively.

\subsection{Optimized transmission boundary operators}

The convergence of the domain decomposition algorithm is fundamentally related to the choice of the operator $\mathcal{S}$. For the time-harmonic Maxwell's equations, the first converging iterative algorithm has been proposed by Després in [10] where a simple impedance boundary operator is proposed

$$
\mathcal{S}^{0}=\mathbf{I} \text {. }
$$

In the sequel, the corresponding zeroth-order Impedance Boundary Condition (IBC) is designated by $\operatorname{IBC}(0)$. A convergence analysis of the DDM method for this boundary condition and for two half-spaces of $\mathbb{R}^{3}$ has been developed in $[12,15]$. The approach, based on Fourier transforms, shows that the algorithm converges only for the propagating modes. For the evanescent modes, the corresponding radius of convergence is equal to 1 , which makes the method stagnates or diverges. Surprisingly enough, we prove in Section 4 that the method converges in the case of a decomposition made of concentric spheres. However, the convergence remains slow for the evanescent modes. To improve the convergence factor for these special modes, Alonso et al. [1] derive an optimized impedance boundary condition by using a Fourier frequency decomposition. They adapt the technique developed by Gander in [17] for the Helmholz equation to get a zero order optimized impedance boundary condition called here $\operatorname{GIBC}(\alpha)$ (GIBC means Generalized Impedance Boundary Condition). For the Maxwell's equation, the $\operatorname{GIBC}(\alpha)$ impedance operator writes down

$$
\mathcal{S}^{\alpha}=\alpha\left(\mathbf{I}-\frac{1}{k^{2}} \operatorname{curl}_{\Sigma_{i j}} \operatorname{curl}_{\Sigma_{i j}}\right)
$$

where $\alpha$ is judiciously chosen thanks to an optimization process (see Section 4.1). The same condition is proposed in [12] for the first-order system of Maxwell's equations. In [29], Peng et al. show that the DDM converges for a well-chosen complex-valued number $\alpha$ and a decomposition into two half-spaces but by considering both the TE (Transverse Electric) and TM (Transverse Magnetic) modes. The improvement of the rate of convergence for the evanescent modes is obtained at the price of the deterioration of the rate of convergence for the propagative modes. To improve this last transmission boundary condition for the two families of modes, Rawat and Lee [30] introduce the following optimized transmission boundary condition by using two second-order operators

$$
\mathcal{S}^{\alpha, \beta}=\left(\mathbf{I}+\frac{\alpha}{k^{2}} \nabla_{\Sigma_{i j}} \operatorname{div}_{\Sigma_{i j}}\right)^{-1}\left(\mathbf{I}-\frac{\beta}{k^{2}} \operatorname{curl}_{\Sigma_{i j}} \operatorname{curl}_{\Sigma_{i j}}\right),
$$


where $\alpha$ and $\beta$ are chosen so that an optimal convergence rate is obtained for the (TE) and (TM) modes. We denote this boundary condition by $\operatorname{GIBC}(\alpha, \beta)$ in the sequel of the paper. In the half-space case, we refer to [30] for the expression of $\alpha$ and $\beta$. Similar boundary conditions are derived in [12] for the first-order Maxwell's equations. Recently, in [11], the authors proved that the convergence rates and the optimization processes for the first- and second-order formulations are finally the same.

When developing optimized DDMs in [7], the authors use highly accurate square-root/Padétype On-Surface Radiation Conditions (OSRCs) [21, 4, 6, 26, 3, 31, 5, 14] as transmission boundary conditions, which are also GIBCs. While being easy-to-use and direct to implement in a finite element environment, these GIBCs lead to the construction of fast converging non-overlapping DDMs, most particularly when computing the solution to high-frequency three-dimensional acoustics scattering problems. In [14], the extension of this high-order OSRC has been developed for the three-dimensional first-order system of Maxwell's equations. When coming back to the secondorder formulation, the corresponding square-root GIBC (that we denote by GIBC $(\mathrm{sq}, \varepsilon)$ ) for the DDM can be written as

$$
\begin{aligned}
\mathcal{S}^{\mathrm{sq}, \varepsilon} & =\boldsymbol{\Lambda}_{1, \varepsilon}^{-1} \boldsymbol{\Lambda}_{2, \varepsilon}, \quad \boldsymbol{\Lambda}_{1, \varepsilon}=\left(\mathbf{I}+\nabla_{\Sigma_{i j}} \frac{1}{k_{\varepsilon}^{2}} \operatorname{div}_{\Sigma_{i j}}-\operatorname{curl}_{\Sigma_{i j}} \frac{1}{k_{\varepsilon}^{2}} \operatorname{curl}_{\Sigma_{i j}}\right)^{1 / 2}, \\
\boldsymbol{\Lambda}_{2, \varepsilon} & =\mathbf{I}-\operatorname{curl}_{\Sigma_{i j}} \frac{1}{k_{\varepsilon}^{2}} \operatorname{curl}_{\Sigma_{i j}},
\end{aligned}
$$

where the complex wavenumber $k_{\varepsilon}$ is defined by: $k_{\varepsilon}=k+\imath \varepsilon$, with the optimal parameter $\varepsilon=$ $0.39 k^{1 / 3} \mathcal{H}_{\Sigma_{i j}}^{2 / 3}$, where $\mathcal{H}_{\Sigma_{i j}}$ is the the local mean curvature on $\Sigma_{i j}$, e.g. the average of the two principal curvatures at the interface. Finally, $A^{1 / 2}$ stands for the square-root of the operator $A$, where the square-root of a complex-valued number $z$ is taken with branch-cut along the negative real axis.

The construction of this GIBC is realized in three steps [14]

1) the half-space case is considered and the construction of the DtN operator is realized by Fourier analysis,

2 ) the extension to a sphere $\mathbb{S}_{R}$ (of radius $R>0$ ) is made by considering the local tangent plane approximation of the DtN map to a spherical surface and a regularization procedure of a square-root operator with optimal damping parameter $\varepsilon$ for $\mathbb{S}_{R}$,

3) and finally the approximation (10) of the MtE operator for a three-dimensional general convex-shaped smooth surface $\Gamma\left(:=\Sigma_{i j}\right.$ in the DDM context $)$ is obtained by considering the local osculating sphere.

A more adapted form of the square-root GIBC defined by (10) is given by

$$
\boldsymbol{\Lambda}_{2, \varepsilon}\left(\gamma_{i}^{T}\left(\mathbf{E}_{i}^{p+1}\right)\right)-\frac{\imath}{k} \boldsymbol{\Lambda}_{1, \varepsilon} \gamma_{i}^{t}\left(\operatorname{curl} \mathbf{E}_{i}^{p+1}\right)=\boldsymbol{\Lambda}_{2, \varepsilon}\left(\gamma_{j}^{T}\left(\mathbf{E}_{j}^{p}\right)\right)+\frac{\imath}{k} \boldsymbol{\Lambda}_{1, \varepsilon} \gamma_{j}^{t}\left(\operatorname{curl} \mathbf{E}_{j}^{p}\right), \quad \text { on } \Gamma=\Sigma_{i j},
$$

and in this case one gets

$$
\mathbf{g}_{j i}^{p+1}=\boldsymbol{\Lambda}_{2, \varepsilon}\left(\gamma_{i}^{T}\left(\mathbf{E}_{i}^{p+1}\right)\right)+\frac{\imath}{k} \boldsymbol{\Lambda}_{1, \varepsilon} \gamma_{i}^{t}\left(\operatorname{curl} \mathbf{E}_{i}^{p+1}\right)=-\mathbf{g}_{i j}^{p}+2 \boldsymbol{\Lambda}_{2, \varepsilon}\left(\gamma_{i}^{T}\left(\mathbf{E}_{i}^{p+1}\right)\right), \quad \text { on } \Sigma_{i j} .
$$

The IBC (7) and the GIBCs (8)-(9) are defined by local surface operators. In contrast, the GIBC given by (10)-(11) is nonlocal because of the presence of the square-root operator.

If we set $k_{\varepsilon}=\alpha^{-1 / 2} k=-\beta^{-1 / 2} k$, let us remark that the Rawat-Lee condition $\operatorname{GIBC}(\alpha,-\alpha)$ with the operator (9) can be seen as $\operatorname{GIBC}(\mathrm{sq}, \varepsilon)$, where $\boldsymbol{\Lambda}_{1, \varepsilon}$ is approximated by 


$$
\boldsymbol{\Lambda}_{1, \varepsilon}=\left(\mathbf{I}+\nabla_{\Sigma_{i j}} \frac{1}{k_{\varepsilon}^{2}} \operatorname{div}_{\Sigma_{i j}}-\operatorname{curl}_{\Sigma_{i j}} \frac{1}{k_{\varepsilon}^{2}} \operatorname{curl}_{\Sigma_{i j}}\right)^{1 / 2} \approx\left(\mathbf{I}+\nabla_{\Sigma_{i j}} \frac{1}{k_{\varepsilon}^{2}} \operatorname{div}_{\Sigma_{i j}}\right)^{1 / 2} \approx \mathbf{I}+\nabla_{\Sigma_{i j}} \frac{1}{k_{\varepsilon}^{2}} \operatorname{div}_{\Sigma_{i j}}
$$

which corresponds to a first-order Taylor expansion of the square-root operator. We will see in what follows that a high-order complex-valued Padé approximation of the full nonlocal operator (11) can be used to get a local representation that is well-suited for a numerical approximation based on finite element methods and leads to quasi-optimal convergence of the DDM.

\section{Convergence analysis for a model problem}

We now study the convergence rate and spectral properties of the DDM algorithm (3) when using the square-root transmission operator. In [12, 30], a Fourier analysis has been performed for computing the convergence factor of the algorithm for each Fourier mode when using (8) or (9). The assumption for the derivation is that the decomposition of $\mathbb{R}^{3}$ uses two half-space subdomains. Here, we consider a more general situation: the whole domain $\Omega=\mathbb{R}^{3}$ is separated in two curved subdomains $\Omega_{1}$ and $\Omega_{2}$ by a spherical boundary of radius $R$

$$
\Omega_{1}=\left\{\mathbf{x} \in \mathbb{R}^{3},\|\mathbf{x}\|>R\right\}, \quad \Omega_{2}=\left\{\mathbf{x} \in \mathbb{R}^{3},\|\mathbf{x}\|<R\right\},
$$

with $\Sigma=\partial \Omega_{1}=\partial \Omega_{2}:=\mathbb{S}_{R}$. Let us study the spectral convergence properties of the iteration operator $\mathcal{A}$ defined by (5) and associated with this domain decomposition.

\subsection{Convergence theorem}

For this decomposition, the DDM algorithm with the square-root GIBC defined by $\mathcal{S}^{\mathrm{sq}, \varepsilon}$ consists in solving, for $p=1,2, \ldots$, the coupled problems in the two subdomains

$$
\left\{\begin{array}{l}
\text { curl curl } \mathbf{E}_{1}^{p+1}-k^{2} \mathbf{E}_{1}^{p+1}=\mathbf{0}, \quad \text { in } \Omega_{1}, \\
\mathbf{g}_{21}^{p+1}:=-\imath k \mathcal{S}^{\mathrm{sq}, \varepsilon}\left(\gamma_{1}^{T}\left(\mathbf{E}_{1}^{p+1}\right)\right)-\gamma_{1}^{t}\left(\operatorname{curl} \mathbf{E}_{1}^{p+1}\right)=-\imath k \mathcal{S}^{\mathrm{sq}, \varepsilon}\left(\gamma_{2}^{T}\left(\mathbf{E}_{2}^{p}\right)\right)+\gamma_{2}^{t}\left(\operatorname{curl} \mathbf{E}_{2}^{p}\right), \quad \text { on } \Sigma,
\end{array}\right.
$$

and

$$
\left\{\begin{array}{l}
\text { curl curl } \mathbf{E}_{2}^{p+1}-k^{2} \mathbf{E}_{2}^{p+1}=\mathbf{0} \quad \text { in } \Omega_{2}, \\
\mathbf{g}_{12}^{p+1}:=-\imath k \mathcal{S}^{\mathrm{sq}, \varepsilon}\left(\gamma_{2}^{T}\left(\mathbf{E}_{2}^{p+1}\right)\right)-\gamma_{2}^{t}\left(\mathbf{c u r l} \mathbf{E}_{2}^{p+1}\right)=-\imath k \mathcal{S}^{\mathrm{sq}, \varepsilon}\left(\gamma_{1}^{T}\left(\mathbf{E}_{1}^{p}\right)\right)+\gamma_{1}^{t}\left(\mathbf{c u r l} \mathbf{E}_{1}^{p}\right), \quad \text { on } \Sigma .
\end{array}\right.
$$

Since we have a spherical transmission boundary, we can explicitly write the solutions to (14) and (15) by using the vectorial spherical harmonics. We then deduce the tangential trace $\gamma_{i}^{t}\left(\mathbf{c u r l} \mathbf{E}_{i}^{p}\right)$ and tangential component trace $\gamma_{i}^{T}\left(\mathbf{E}_{i}^{p}\right)$

$$
\begin{aligned}
& \gamma_{1}^{T}\left(\mathbf{E}_{1}^{p}\right)=\frac{1}{k} \sum_{m=1}^{\infty} \imath \alpha_{m}^{p} \xi_{m}^{(1)^{\prime}}(k R) \nabla_{\mathbb{S}_{R}} Y_{m}^{c, 1}(\theta, \phi)-\frac{1}{k} \sum_{m=1}^{\infty} \beta_{m}^{p} \xi_{m}^{(1)}(k R) \mathbf{n} \times \nabla_{\mathbb{S}_{R}} Y_{m}^{s, 1}(\theta, \phi), \\
& \gamma_{1}^{t}\left(\mathbf{c u r l} \mathbf{E}_{1}^{p}\right)=\frac{1}{k} \sum_{m=1}^{\infty} \alpha_{m}^{p} \xi_{m}^{(1)}(k R) \nabla_{\mathbb{S}_{R}} Y_{m}^{c, 1}(\theta, \phi)-\frac{1}{k} \sum_{m=1}^{\infty} \imath \beta_{m}^{p} \xi_{m}^{(1)^{\prime}}(k R) \mathbf{n} \times \nabla_{\mathbb{S}_{R}} Y_{m}^{s, 1}(\theta, \phi),
\end{aligned}
$$

and

$$
\begin{aligned}
\gamma_{2}^{T}\left(\mathbf{E}_{2}^{p}\right) & =\frac{1}{k} \sum_{m=1}^{\infty} \imath \gamma_{m}^{p} \psi_{m}^{\prime}(k R) \nabla_{\mathbb{S}_{R}} Y_{m}^{c, 1}(\theta, \phi)-\frac{1}{k} \sum_{m=1}^{\infty} \delta_{m}^{p} \psi_{m}(k R) \mathbf{n} \times \nabla_{\mathbb{S}_{R}} Y_{m}^{s, 1}(\theta, \phi), \\
\gamma_{2}^{t}\left(\operatorname{curl} \mathbf{E}_{2}^{p}\right) & =\frac{1}{k} \sum_{m=1}^{\infty} \gamma_{m}^{p} \psi_{m}(k R) \nabla_{\mathbb{S}_{R}} Y_{m}^{c, 1}(\theta, \phi)-\frac{1}{k} \sum_{m=1}^{\infty} \imath \delta_{m}^{p} \psi_{m}^{\prime}(k R) \mathbf{n} \times \nabla_{\mathbb{S}_{R}} Y_{m}^{s, 1}(\theta, \phi) .
\end{aligned}
$$

Functions $\psi_{m}$ and $\zeta_{m}$ are respectively the first- and second-kind Ricatti-Bessel functions of order $m, \xi_{m}^{(1)}=\psi_{m}+\imath \zeta_{m}$ is the first-kind spherical Hankel's function of order $m, Y_{m}^{c, 1}(\theta, \phi)=$ 
$P_{m}^{1}(\cos (\theta)) \cos (\phi)$ and $Y_{m}^{s, 1}(\theta, \phi)=P_{m}^{1}(\cos (\theta)) \sin (\phi)$ are the spherical harmonics, $P_{m}^{1}$ being the first-order Legendre function of degree $m$. The operator $\nabla_{\mathbb{S}_{R}}$ is the surface gradient and $(\theta, \phi)$ are the spherical coordinates. We refer to [2] for more details concerning these special functions. The coefficient $\alpha_{m}^{p}, \beta_{m}^{p}, \gamma_{m}^{p}$ and $\delta_{m}^{p}$ are determined by using the transmission boundary conditions in (14)-(15). The expressions (16)-(17) lead to the following equalities

$$
\left\{\begin{aligned}
\mathbf{g}_{21}^{p+1} & :=\sum_{m=1}^{\infty}\left(\mathbf{g}_{21}^{p+1, m}\right)_{1} \nabla_{\mathbb{S}_{R}} Y_{m}^{c, 1}(\theta, \phi)+\sum_{m=1}^{\infty}\left(\mathbf{g}_{21}^{p+1, m}\right)_{2} \mathbf{n} \times \nabla_{\mathbb{S}_{R}} Y_{m}^{s, 1}(\theta, \phi) \\
& =\frac{1}{k} \sum_{m=1}^{\infty} A_{m, 1} \alpha_{m}^{p+1} \nabla_{\mathbb{S}_{R}} Y_{m}^{c, 1}(\theta, \phi)+\frac{1}{k} \sum_{m=1}^{\infty} A_{m, 2} \beta_{m}^{p+1} \mathbf{n} \times \nabla_{\mathbb{S}_{R}} Y_{m}^{s, 1}(\theta, \phi) \\
& =\frac{1}{k} \sum_{m=1}^{\infty} B_{m, 1} \gamma_{m}^{p} \nabla_{\mathbb{S}_{R}} Y_{m}^{c, 1}(\theta, \phi)+\frac{1}{k} \sum_{m=1}^{\infty} B_{m, 2} \delta_{m}^{p} \mathbf{n} \times \nabla_{\mathbb{S}_{R}} Y_{m}^{s, 1}(\theta, \phi), \\
\mathbf{g}_{12}^{p+1} & :=\sum_{m=1}^{\infty}\left(\mathbf{g}_{12}^{p+1, m}\right)_{1} \nabla_{\mathbb{S}_{R}} Y_{m}^{c, 1}(\theta, \phi)+\sum_{m=1}^{\infty}\left(\mathbf{g}_{12}^{p+1, m}\right)_{2} \mathbf{n} \times \nabla_{\mathbb{S}_{R}} Y_{m}^{s, 1}(\theta, \phi) \\
& =\frac{1}{k} \sum_{m=1}^{\infty} A_{m, 3} \gamma_{m}^{p+1} \nabla_{\mathbb{S}_{R}} Y_{m}^{c, 1}(\theta, \phi)-\frac{1}{k} \sum_{m=1}^{\infty} A_{m, 4} \delta_{m}^{p+1} \mathbf{n} \times \nabla_{\mathbb{S}_{R}} Y_{m}^{s, 1}(\theta, \phi) \\
& =\frac{1}{k} \sum_{m=1}^{\infty} B_{m, 3} \alpha_{m}^{p} \nabla_{\mathbb{S}_{R}} Y_{m}^{c, 1}(\theta, \phi)-\frac{1}{k} \sum_{m=1}^{\infty} B_{m, 4} \beta_{m}^{p} \mathbf{n} \times \nabla_{\mathbb{S}_{R}} Y_{m}^{s, 1}(\theta, \phi),
\end{aligned}\right.
$$

where

$$
\begin{cases}A_{m, 1}=\imath \mu_{m, \varepsilon}^{-\frac{1}{2}} \xi_{m}^{(1)^{\prime}}(k R)-\xi_{m}^{(1)}(k R), & B_{m, 1}=\imath \mu_{m, \varepsilon}^{-\frac{1}{2}} \psi_{m}^{\prime}(k R)-\psi_{m}(k R), \\ A_{m, 2}=\imath \xi_{m}^{(1)^{\prime}}(k R)-\mu_{m, \varepsilon}^{\frac{1}{2}} \xi_{m}^{(1)}(k R), & B_{m, 2}=\imath \psi_{m}^{\prime}(k R)-\mu_{m, \varepsilon}^{\frac{1}{2}} \psi_{m}(k R), \\ A_{m, 3}=\imath \mu_{m, \varepsilon}^{-\frac{1}{2}} \psi_{m}^{\prime}(k R)+\psi_{m}(k R), & B_{m, 3}=\imath \mu_{m, \varepsilon}^{-\frac{1}{2}} \xi_{m}^{(1)^{\prime}}(k R)+\xi_{m}^{(1)}(k R), \\ A_{m, 4}=\imath \psi_{m}^{\prime}(k R)+\mu_{m, \varepsilon}^{\frac{1}{2}} \psi_{m}(k R), & B_{m, 4}=\imath \xi_{m}^{(1)^{\prime}}(k R)+\mu_{m, \varepsilon}^{\frac{1}{2}} \xi_{m}^{(1)}(k R),\end{cases}
$$

setting

$$
\mu_{m, \varepsilon}=1-\frac{m(m+1)}{k_{\varepsilon}^{2} R^{2}}=\frac{R^{2}\left(k^{2}+\varepsilon^{2}\right)^{2}-m(m+1)\left(k^{2}+\varepsilon^{2}\right)}{R^{2}\left(k^{2}+\varepsilon^{2}\right)^{2}}+2 \frac{m(m+1) \varepsilon}{R^{2}\left(k^{2}+\varepsilon^{2}\right)^{2}} l .
$$

To obtain the expressions (18)-(19), we used the following properties of the spherical harmonics (see [27], p. 39):

$$
\left\{\begin{array}{l}
\boldsymbol{\Delta}_{\mathbb{S}_{R}} \nabla_{\mathbb{S}_{R}} Y_{m}^{n}=-\frac{m(m+1)}{R^{2}} \nabla_{\mathbb{S}_{R}} Y_{m}^{n} \\
\boldsymbol{\Delta}_{\mathbb{S}_{R}} \operatorname{curl}_{\mathbb{S}_{R}} Y_{m}^{n}=-\frac{m(m+1)}{R^{2}} \operatorname{curl}_{\mathbb{S}_{R}} Y_{m}^{n}
\end{array}\right.
$$

where $Y_{m}^{n}$ is the spherical harmonics of order $(m, n)$. Since $\left(\nabla_{\mathbb{S}_{R}} Y_{m}^{n}, \mathbf{n} \times \nabla_{\mathbb{S}_{R}} Y_{m}^{n}\right)$ is a basis of the space of tangent vector fields on $\mathbb{S}_{R}$, we have

$$
\left\{\begin{array}{cl}
\left(\mathbf{g}_{12}^{p+1, m}\right)_{1}=\frac{A_{m, 1}}{k} \alpha_{m}^{p+1}=\frac{B_{m, 1}}{k} \gamma_{m}^{p}, & \left(\mathbf{g}_{12}^{p+1, m}\right)_{2}=\frac{A_{m, 2}}{k} \beta_{m}^{p+1}=\frac{B_{m, 2}}{k} \delta_{m}^{p} \\
\left(\mathbf{g}_{21}^{p+1, m}\right)_{1}=\frac{A_{m, 3}}{k} \gamma_{m}^{p+1}=\frac{B_{m, 3}}{k} \alpha_{m}^{p}, & \left(\mathbf{g}_{21}^{p+1, m}\right)_{2}=\frac{A_{m, 4}}{k} \delta_{m}^{p+1}=\frac{B_{m, 4}}{k} \beta_{m}^{p} .
\end{array}\right.
$$

From (21), we obtain

$$
\alpha_{m}^{p+1}=\frac{B_{m, 1}}{A_{m, 1}} \gamma_{m}^{p}, \quad \beta_{m}^{p+1}=\frac{B_{m, 2}}{A_{m, 2}} \delta_{m}^{p}, \quad \gamma_{m}^{p+1}=\frac{B_{m, 3}}{A_{m, 3}} \alpha_{m}^{p}, \quad \delta_{m}^{p+1}=\frac{B_{m, 4}}{A_{m, 4}} \beta_{m}^{p},
$$


implying that

$$
\left\{\begin{array}{l}
\left(\mathbf{g}_{21}^{p+1, m}\right)_{1}=\frac{B_{m, 1}}{A_{m, 3}} \frac{A_{m, 3}}{k} \gamma_{m}^{p}=\frac{B_{m, 1}}{A_{m, 3}}\left(\mathbf{g}_{12}^{p, m}\right)_{1}, \quad\left(\mathbf{g}_{21}^{p+1, m}\right)_{2}=\frac{B_{m, 2}}{A_{m, 4}} \frac{A_{m, 4}}{k} \delta_{m}^{p}=\frac{B_{m, 2}}{A_{m, 4}}\left(\mathbf{g}_{12}^{p, m}\right)_{2}, \\
\left(\mathbf{g}_{12}^{p+1, m}\right)_{1}=\frac{B_{m, 3}}{A_{m, 1}} \frac{A_{m, 1}}{k} \alpha_{m}^{p}=\frac{B_{m, 3}}{A_{m, 1}}\left(\mathbf{g}_{21}^{p, m}\right)_{1}, \quad\left(\mathbf{g}_{12}^{p+1, m}\right)_{2}=\frac{B_{m, 4}}{A_{m, 2}} \frac{A_{m, 2}}{k} \beta_{m}^{p}=\frac{B_{m, 4}}{A_{m, 2}}\left(\mathbf{g}_{21}^{p, m}\right)_{2} .
\end{array}\right.
$$

Finally, we obtain the following iterative scheme

$$
\mathbf{g}^{p+1, m}=\left(\begin{array}{l}
\left(\mathbf{g}_{21}^{p+1, m}\right)_{1} \\
\left(\mathbf{g}_{21}^{p+1, m}\right)_{2} \\
\left(\mathbf{g}_{12}^{p+1, m}\right)_{1} \\
\left(\mathbf{g}_{12}^{p+1, m}\right)_{2}
\end{array}\right)=\mathbb{A}_{m} \mathbf{g}^{p, m}:=\left(\begin{array}{cccc}
0 & 0 & \frac{B_{m, 1}}{A_{m, 3}} & 0 \\
0 & 0 & 0 & \frac{B_{m, 2}}{A_{m, 4}} \\
\frac{B_{m, 3}}{A_{m, 1}} & 0 & 0 & 0 \\
0 & \frac{B_{m, 4}}{A_{m, 2}} & 0 & 0
\end{array}\right) \mathbf{g}^{p, m},
$$

with $\mathbb{A}_{m}$ the iteration matrix for a mode $m \geq 1$. Since our problem is linear and we have an orthonormal decomposition of the iteration operator $\mathcal{A}$ on the spherical harmonics basis, we can prove that $\mathcal{A}=\operatorname{diag}\left(\left(\mathbb{A}_{m}\right)_{m \geq 1}\right)$. Therefore, studying the global convergence of the two spherical $\mathrm{DDM}$ for $\mathcal{A}$ requires the spectral study of the modal iteration matrices $\mathbb{A}_{m}$, for $m \geq 1$.

For a fixed-point (Jacobi-type) method and a mode $m$, the convergence rate of the iterative method is given by the spectral radius $\rho\left(\mathbb{A}_{m}\right)$ of $\mathbb{A}_{m}$. A simple calculation shows that the eigenvalues of $\mathbb{A}_{m}$ are

$$
\lambda_{m, 1}=\sqrt{\frac{B_{m, 1} B_{m, 3}}{A_{m, 1} A_{m, 3}}}=-\lambda_{m, 2}, \quad \lambda_{m, 3}=\sqrt{\frac{B_{m, 2} B_{m, 4}}{A_{m, 2} A_{m, 4}}}=-\lambda_{m, 4} .
$$

Let us remark that $\lambda_{m, 1}=\lambda_{m, 3}$. The global iterative method (14)-(15) converges if and only if the spectral radius $\rho=\max _{m \geq 1} \rho\left(\mathbb{A}_{m}\right)$ is strictly less than one. The result is embedded in the following Theorem.

Theorem 1. For any given initial guess $\mathbf{E}_{1}^{0} \in\left(L^{2}\left(\Omega_{1}\right)\right)^{3}$ and $\mathbf{E}_{2}^{0} \in\left(L^{2}\left(\Omega_{2}\right)\right)^{3}$, the iterative DDM (14)-(15) converges in $\left(L^{2}\left(\Omega_{1}\right)\right)^{3} \times\left(L^{2}\left(\Omega_{2}\right)\right)^{3}$, i.e the convergence factor

$$
\forall m \geq 1, \quad \rho\left(\mathbb{A}_{m}\right)=\left|\sqrt{\frac{B_{m, 2} B_{m, 4}}{A_{m, 2} A_{m, 4}}}\right|=\left|\sqrt{\mathcal{T}_{m, 1} \mathcal{T}_{m, 2}}\right|<1 .
$$

In the above expressions, we set

$$
\mathcal{T}_{m, 1}=\frac{\mathcal{Z}_{m, 1}-\imath \mu_{m, \varepsilon}^{\frac{1}{2}}}{\mathcal{Z}_{m, 1}+\imath \mu_{m, \varepsilon}^{\frac{1}{2}}}, \quad \mathcal{T}_{m, 2}=\frac{\mathcal{Z}_{m, 2}+\imath \mu_{m, \varepsilon}^{\frac{1}{2}}}{\mathcal{Z}_{m, 2}-\imath \mu_{m, \varepsilon}^{\frac{1}{2}}}
$$

with $\mathcal{Z}_{m, 1}=\frac{\xi_{m}^{(1)^{\prime}}(k R)}{\xi_{m}^{(1)}(k R)}$ and $\mathcal{Z}_{m, 2}=\frac{\psi_{m}^{\prime}(k R)}{\psi_{m}(k R)}$

To prove Theorem 1, let us first consider the following proposition.

Proposition 1. For $\varepsilon>0$ and any $m \geq 1$, we have,

$$
\left|\mathcal{T}_{m, 1}\right|<1
$$

If $\varepsilon=0$ and $m \neq \frac{\sqrt{1+4 R^{2} k^{2}}-1}{2}:=m_{t}$, then the inequality (25) also holds. 
Proof. Setting $\mathcal{Z}_{m, 1}=x_{m}+\imath y_{m}$ and $\mu_{m, \varepsilon}^{\frac{1}{2}}=\sqrt{r_{m}+\imath s_{m}}=a_{m}+\imath b_{m}$, we obtain

$$
\left|\mathcal{T}_{m, 1}\right|^{2}=\left|\frac{x_{m}+\imath y_{m}-\imath\left(a_{m}+\imath b_{m}\right)}{x_{m}+\imath y_{m}+\imath\left(a_{m}+\imath b_{m}\right)}\right|^{2}=\frac{\left(x_{m}+b_{m}\right)^{2}+\left(y_{m}-a_{m}\right)^{2}}{\left(x_{m}-b_{m}\right)^{2}+\left(y_{m}+a_{m}\right)^{2}},
$$

with

$$
a_{m}=\sqrt{\frac{\sqrt{r_{m}^{2}+s_{m}^{2}}+r_{m}}{2}}>0, \quad b_{m}=\operatorname{sign}\left(s_{m}\right) \sqrt{\frac{\sqrt{r_{m}^{2}+s_{m}^{2}}-r_{m}}{2}}>0
$$

(denoting by sign the sign function).

If $\varepsilon>0$ and $x_{m}<0<y_{m}$, we have from (26): $\left|\mathcal{T}_{m, 1}\right|^{2}<1$, for all $m$. Let us prove that $x_{m}<0<y_{m}$. Indeed, by writing $\mathcal{Z}_{m, 1}$ as

$$
\mathcal{Z}_{m, 1}=\frac{\xi_{m}^{(1)^{\prime}}(k R)}{\xi_{m}^{(1)}(k R)}=\frac{\xi_{m}^{(1)^{\prime}}(k R) \overline{\xi_{m}^{(1)}(k R)}}{\left|\xi_{m}^{(1)}(k R)\right|^{2}}
$$

we have [2]

$$
y_{m}=\Im\left(\xi_{m}^{(1)^{\prime}}(k R) \overline{\xi_{m}^{(1)}(k R)}\right)=\psi_{m}(k R) \zeta_{m}^{\prime}(k R)-\psi_{m}^{\prime}(k R) \zeta_{m}(k R)=1>0 .
$$

Furthermore, we obtain

$$
x_{m}=\Re\left(\xi_{m}^{(1)^{\prime}}(k R) \overline{\xi_{m}^{(1)}(k R)}\right)=\frac{1}{2}\left(\left|\xi_{m}^{(1)}(k R)\right|^{2}\right)^{\prime} .
$$

To prove that $x_{m}<0$, we use the property [32] that $\left|H_{\nu}^{(1)}(t)\right|^{2}$ is strictly decreasing with respect to the positive variable $t$, for any fixed real-value $\nu$. We have

$$
x_{m}=\left(\left|\xi_{m}^{(1)}(t)\right|^{2}\right)^{\prime}=\left(\frac{\pi}{2 t}\left(\left|H_{m+1 / 2}^{(1)}(t)\right|^{2}\right)^{\prime}=\left(\frac{\pi}{2 t}\right)^{\prime}\left|H_{m+1 / 2}^{(1)}(t)\right|^{2}+\left(\frac{\pi}{2 t}\right)\left(\left|H_{m+1 / 2}^{(1)}(t)\right|^{2}\right)^{\prime}<0 .\right.
$$

Let us now assume that $\varepsilon=0$. If one has a propagating mode, i.e. $1 \leq m<m_{t}$, then $\mu_{m, \varepsilon}^{1 / 2}=a_{m}>0$. For the evanescent modes such that $m>m_{t}, \mu_{m, \varepsilon}^{1 / 2}=\imath b_{m}$, with $b_{m}>0$. In these two cases, we have as previously: $\left|\mathcal{T}_{m, 1}\right|^{2}<1$.

Proposition 2. For $\varepsilon \geq 0$ and any $m>m_{t}$, we have,

$$
\left|\mathcal{T}_{m, 2}\right|<1 .
$$

If $\varepsilon=0$ and $m \leq m_{t}$, the following equality holds:

$$
\left|\mathcal{T}_{m, 2}\right|=1
$$

Proof. The quantity $\mathcal{Z}_{m, 2}$ is real-valued. Therefore, we have

$$
\left|\mathcal{T}_{m, 2}\right|^{2}=\left|\frac{\mathcal{Z}_{m, 2}+\imath\left(a_{m}+\imath b_{m}\right)}{\mathcal{Z}_{m, 2}-\imath\left(a_{m}+\imath b_{m}\right)}\right|^{2}=\frac{\left(\mathcal{Z}_{m, 2}-b_{m}\right)^{2}+a_{m}^{2}}{\left(\mathcal{Z}_{m, 2}+b_{m}\right)^{2}+a_{m}^{2}} .
$$

Let us first consider the case: $\varepsilon \geq 0$ and $m>m_{t}$. Then, we can prove that $\left|\mathcal{T}_{m, 2}\right|^{2}<1$ if one gets $\mathcal{Z}_{m, 2}>0$. Let us write

$$
\mathcal{Z}_{m, 2}=\frac{\psi_{m}^{\prime}(k R)}{\psi_{m}(k R)}=\frac{\psi_{m}^{\prime}(k R) \psi_{m}(k R)}{\psi_{m}^{2}(k R)}
$$


To prove that $\psi_{m}^{\prime}(k R) \psi_{m}(k R)>0$, we use the definition of the functions $\psi_{m}$. For $r \neq 0, \psi_{m}(r)$ is the solution of the ODE

$$
\psi_{m}^{\prime \prime}(r)+\left(1-\frac{m(m+1)}{r^{2}}\right) \psi_{m}(r)=0 .
$$

If we multiply this equation by $\psi_{m}(r)$ and integrate on $(0, k R)$, we get (since $\left.\psi_{m}(0)=0\right)$

$$
\psi_{m}^{\prime}(k R) \psi_{m}(k R)=\int_{0}^{k R}\left(\frac{m(m+1)}{r^{2}}-1\right) \psi_{m}^{2}(r) d r+\int_{0}^{k R} \psi_{m}^{\prime 2}(k R) d r .
$$

If $\left(\frac{m(m+1)}{k^{2} R^{2}}-1\right)>0$, i.e $m>m_{t}$, then $\left(\frac{m(m+1)}{r^{2}}-1\right)>0, \forall r \in(0, k R)$. As a conclusion, this proves that $\psi_{m}^{\prime}(k R) \psi_{m}(k R)>0$ and finally $\left|\mathcal{T}_{m, 2}\right|<1$ by using $(30)$.

Let us now assume that $\varepsilon=0$ and $m \leq m_{t}$. Then, we have (see Proposition 1) $b_{m}=0$ and $\left|\mathcal{T}_{m, 2}\right|^{2}=1$.

Theorem 2. For $\varepsilon=0$ and if $m$ is not a cut-off mode, i.e $m \neq m_{t}$, we have

$$
\left|\mathcal{T}_{m, 1} \mathcal{T}_{m, 2}\right|<1
$$

If $\varepsilon>0$, then there exists $\varepsilon_{\max }$ such that the inequality (32) is satisfied for $0<\varepsilon<\varepsilon_{\max }$.

Proof. If $\varepsilon=0$ and $m \neq m_{t}$, the inequality (32) is a direct consequence of Propositions 1 and 2. For $\varepsilon>0$, the inequality (32) holds for the evanescent modes $\left(m>m_{t}\right)$. For the finite number of propagating modes $\left(1 \leq m \leq m_{t}\right)$ and since (32) holds for $\varepsilon=0$, a continuity argument on $\varepsilon$ proves that there exists $\varepsilon_{\max }$ such that (32) holds for $0<\varepsilon<\varepsilon_{\max }$.

A consequence of this Theorem is that (if $\varepsilon>0$ )

$$
\forall m>1, \rho\left(\mathbb{A}_{m}\right):=\left|\sqrt{\mathcal{T}_{m, 1} \mathcal{T}_{m, 2}}\right|<1,
$$

and therefore the DDM converges globally since the radius of convergence of the iteration matrix is such that $\rho(\mathcal{A}):=\max _{m>1} \rho\left(\mathbb{A}_{m}\right)<1$ for the spherical decomposition. This proves the convergence Theorem 1. Considering $\varepsilon>0$ ensures the well-posedness of the DDM. Finally, $\varepsilon>0$ improves the convergence of the iterative scheme for the transition zone between the propagating and evanescent modes.

Remark 1. If one considers the Després impedance boundary condition IBC(0) (see Eq. (7) which defines $\mathcal{S}^{0}$ ), the convergence factor is then given by

$$
\rho\left(\mathbb{A}_{m}\right)=\left|\sqrt{\frac{\mathcal{Z}_{m, 1}-\imath}{\mathcal{Z}_{m, 1}+\imath} \frac{\mathcal{Z}_{m, 2}+\imath}{\mathcal{Z}_{m, 2}-\imath}}\right| .
$$

As previously, it can be proved that: $\rho\left(\mathbb{A}_{m}\right)<1$ for all $m \geq 1$, i.e that, for a spherical two-domain decomposition, the DDM with Després condition converges. This is a very interesting result since it can be proved [12] that the convergence factor is exactly 1 for the evanescent modes in the case of a two half-space decomposition.

To illustrate the rate of convergence of the DDM with different impedance boundary conditions, let us consider the case of a sphere of radius $R=1$ and for $k=6 \pi$. The maximal number of modes is $m^{\max }=[10 k R]$ (where $[10 k R]$ designates the integer part of $10 k R$ ). We report on Figure 1 the modal spectral radius $\rho\left(\mathbb{A}_{m}\right)$ for the following transmission boundary conditions: $\operatorname{IBC}(0)$ (Eq. $(7)), \operatorname{GIBC}(\alpha)($ Eq. $(8)), \operatorname{GIBC}(\alpha, \beta)(\mathrm{Eq} .(9))$ and $\operatorname{GIBC}(\mathrm{sq}, \varepsilon)($ Eq. (11)). For $\operatorname{GIBC}(\alpha)$ and 


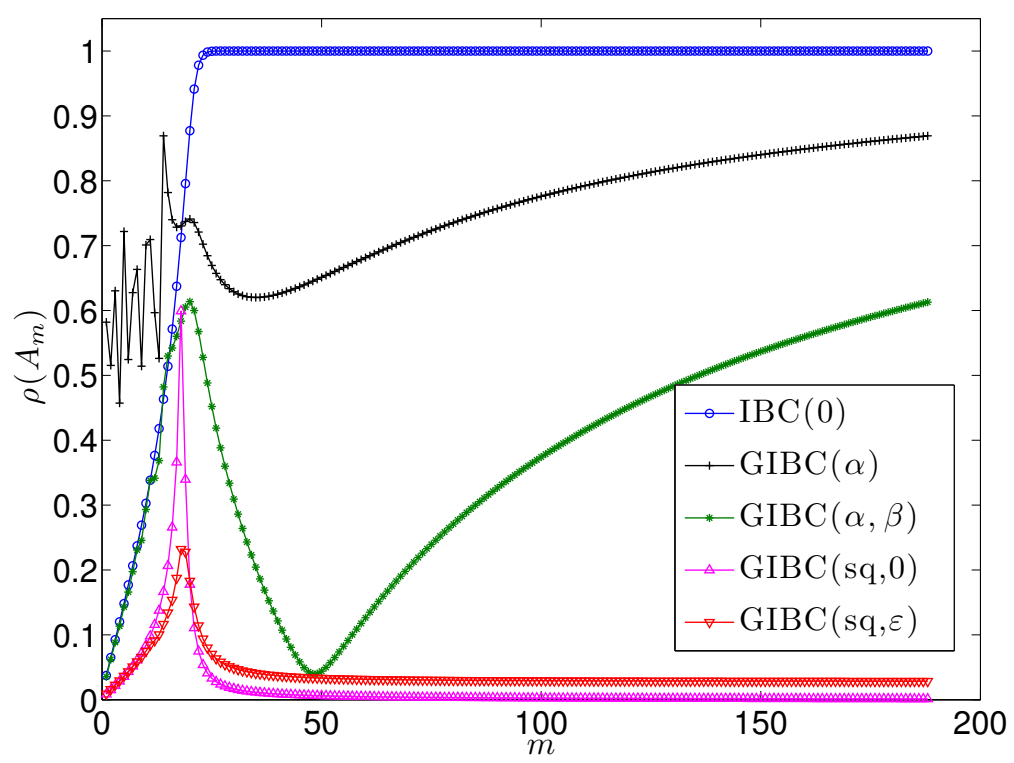

Figure 1: Convergence rate with respect to $m$.

$\operatorname{GIBC}(\alpha, \beta)$, the optimal parameters $\alpha$ and $\beta$ are numerically computed by solving the min-max problem

$$
\min _{(\alpha, \beta) \in \mathbb{C}^{2}} \max _{m \geq 1} \rho\left(\mathbb{A}_{m}\right)
$$

with the Matlab function fminsearch. Let us remark that solving (35) analytically is extremely complicated. A practical alternative consists in using $\operatorname{GIBC}(\alpha)$ and $\operatorname{GIBC}(\alpha, \beta)$ that can be explicitly designed for the half-space approximation. More precisely, $\operatorname{GBC}\left(\alpha_{0}\right)$ is given [1] by considering the optimal parameter

$$
\alpha_{0}=\frac{e^{-\imath \frac{\pi}{4}}}{\left(\left(k^{2}-k_{-}^{2}\right)\left(\xi_{\max }^{2}-k^{2}\right)\right)^{1 / 4}},
$$

with $k^{-}:=k-\pi / L$ and $\xi_{\max }$ the highest spatial Fourier frequency, $L$ being the largest dimension of the transmitting interface. In the case of a spherical interface and according to our notations, we take: $L=2 \pi R$ and $\xi_{\max }=m^{\max }$. For $\operatorname{GIBC}\left(\alpha_{1}, \beta_{1}\right)$ [30], we have

$$
\alpha_{1}=\frac{k}{k+\imath \sqrt{\xi_{\max }^{\mathrm{te}, 2}-k^{2}}}, \quad \beta_{1}=-\frac{k}{k+\imath \sqrt{\xi_{\max }^{\mathrm{tm}, 2}-k^{2}}},
$$

where various values of $\xi_{\max }^{\mathrm{te}}$ and $\xi_{\max }^{\mathrm{tm}}$ are tested. The extension to a spherical interface is nontrivial. Here, we fix the choice to $\xi_{\max }^{\mathrm{te}, \mathrm{tm}}=2 k$ which is numerically optimal for the sphere.

As we remarked above, $\operatorname{IBC}(0)$ leads to $\rho\left(\mathbb{A}_{m}\right)<1$ for the propagative modes $\left(1 \leq m \leq m_{t}\right.$, with $\left.m_{t}=[k R]=18\right)$ but $\rho\left(\mathbb{A}_{m}\right)$ is very close to 1 for the evanescent modes $\left(m>m_{t}\right)$, which results in a globally slowly converging $\operatorname{DDM}$. For $\operatorname{GIBC}(\alpha)$, we see that $\rho\left(\mathbb{A}_{m}\right)<1$, for all $m$. This is still improved for $\operatorname{GIBC}(\alpha, \beta)$. $\operatorname{GIBC}(\mathrm{sq}, 0)$ leads to a better convergence rate which can furthermore be optimized in the transition zone by using $\operatorname{GIBC}(\mathrm{sq}, \varepsilon)$ (with the value parameter $\varepsilon=$ $\left.0.39 k^{1 / 3} R^{-2 / 3}\right)$. Finally, a numerical study shows that $\operatorname{GIBC}\left(\alpha_{0}\right)$ or $\operatorname{GIBC}\left(\alpha_{1}, \beta_{1}\right)$ with formulas (36)-(37) generally lead to a spectral radius larger than one, whatever the values of $k^{-}, \xi_{\max }$ and $\xi_{\max }^{\mathrm{te}, \mathrm{tm}}$. 


\subsection{Quasi-optimality - iterative Krylov subspace solvers}

The aim of this Section is to show that the DDM is quasi-optimal in the sense that there exists an accumulation point of the eigenvalues in the complex plane for the evanescent modes, i.e. when $m \gg k R$. The result is stated asymptotically for the spherical DDM. Let us recall that

$$
H_{m+1 / 2}^{(1)}(t) \approx-\imath \frac{\Gamma_{0}(m+1 / 2)}{\pi} \frac{2^{m}}{t^{m+1 / 2}}, \quad J_{m+1 / 2}(t) \approx-\frac{1}{\Gamma_{0}(m+3 / 2)} \frac{t^{m+1 / 2}}{2^{m+1 / 2}},
$$

for $|m+3 / 2| \gg t>0$, where $\Gamma_{0}$ is the Gamma function. By using the expression of the Gamma function for half integers and the definition of $\xi_{m}$ and $\psi_{m}$, one gets the asymptotic approximations

$$
\xi_{m}(t) \approx-\imath \frac{(2 m) !}{m !} \frac{2^{-m}}{t^{m}}, \quad \psi_{m}(t) \approx \frac{(m+1) !}{(2 m+2) !} 2^{m+1} t^{m+1} .
$$

Now, by using the expressions of $\mathcal{Z}_{m, 1}$ and $\mathcal{Z}_{m, 2}$ given in Theorem 1 , we obtain

$$
\mathcal{Z}_{m, 1} \approx-\frac{m}{k R}, \quad \mathcal{Z}_{m, 2} \approx-\frac{m+1}{k R},
$$

for the evanescent modes. Finally, we deduce that

$$
\lim _{m \rightarrow \infty} \lambda_{m,(1,3)}=-\lim _{m \rightarrow \infty} \lambda_{m,(2,4)}=\frac{\imath \varepsilon}{2 k+\imath \varepsilon} .
$$

This means that we have two opposite accumulation points in the complex plane for the evanescent modes.

In a practical DDM, Krylov subspace solvers are generally preferred to a Jacobi (fixed-point) method since they are more robust and converge faster. This is most particularly true when there is a clustering of the eigenvalues in the complex plane. When using the DDM with GIBC(sq, $\varepsilon$ ), we just have seen above that this is the case when solving: $(\mathcal{I}-\mathcal{A}) \mathbf{g}=\mathbf{b}$, for a spherical domain decomposition. Indeed, in this situation, the eigenvalues of $(\mathcal{I}-\mathcal{A})$ are given by $1-\lambda_{m,(1,2,3,4)}$, for $m \geq 1$, and satisfy

$$
\lim _{m \rightarrow \infty} 1-\lambda_{m,(1,3)}=1-\frac{\imath \varepsilon}{2 k+\imath \varepsilon}, \quad \quad \lim _{m \rightarrow \infty} 1-\lambda_{m,(2,4)}=1+\frac{\imath \varepsilon}{2 k+\imath \varepsilon} .
$$

To illustrate this claim, let us consider the same parameters as in the numerical situation of Section 4.1. We compare on Figure 2 the history of the residual with respect to the number of iterations \#iter for the Jacobi and GMRES solvers to converge with a stopping criterion equal to $10^{-8}$, and for the various transmission boundary conditions. As we can observe, there is a hierarchy in the convergence curves that is directly connected to the increasing order of the GIBCs, the best convergence being obtained for $\operatorname{GIBC}(\mathrm{sq}, \varepsilon)$. When using $\operatorname{GIBC}\left(\alpha_{1}, \beta_{1}\right)$ with the parameters given in (37), the number of iterations is about the same as for $\operatorname{GIBC}\left(\alpha_{0}\right)$. Finally, let us remark that some tests show that the Jacobi method does not converge for all the GIBCs except for GIBC(sq, 0$)$ and $\operatorname{GIBC}(\mathrm{sq}, \varepsilon)$.

\section{Localization of the square-root GIBC}

The square-root transmission boundary condition, given by (10)-(11), is nonlocal since it is defined by the pseudodifferential operator $\boldsymbol{\Lambda}_{1, \varepsilon}$

$$
\mathbf{\Lambda}_{1, \varepsilon}:=(\mathbf{I}+\mathscr{T})^{1 / 2}
$$

setting

$$
\mathscr{T}:=\nabla_{\Gamma} \frac{1}{k_{\varepsilon}^{2}} \operatorname{div}_{\Gamma}-\operatorname{curl}_{\Gamma} \frac{1}{k_{\varepsilon}^{2}} \operatorname{curl}_{\Gamma}
$$




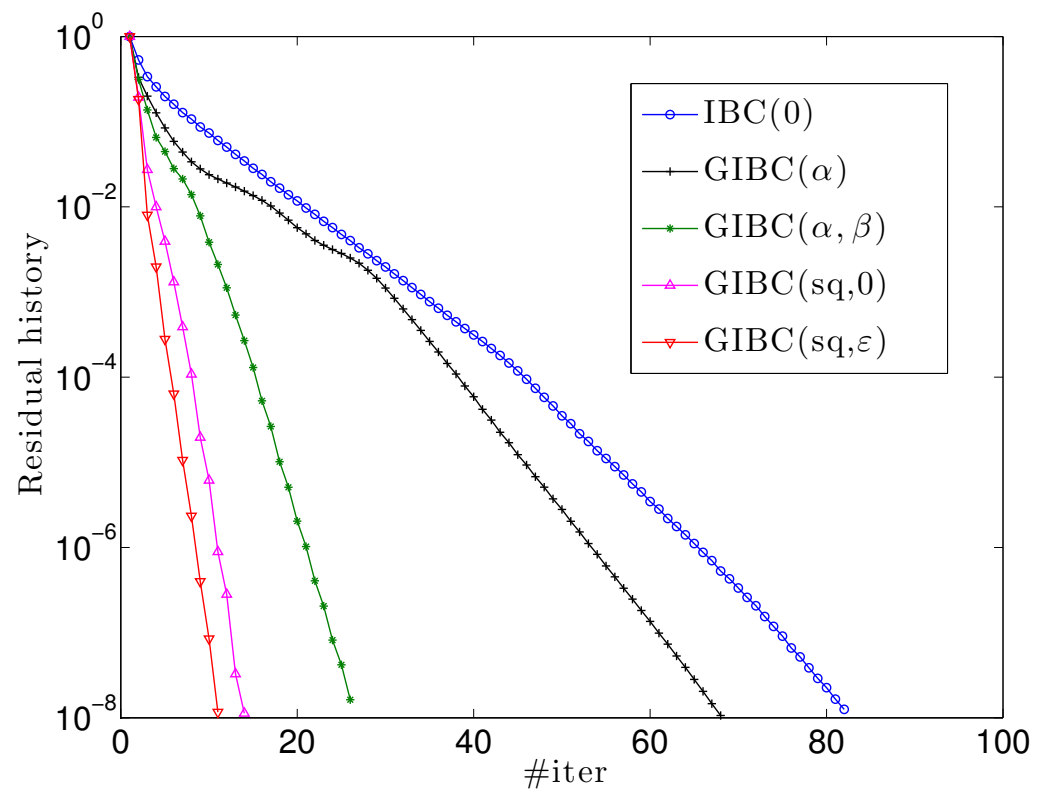

Figure 2: Residual history of GMRES vs. \#iter for the various GIBCs.

Such an operator is impracticable in a finite element context since it generates a full matrix part associated with the transmitting boundary. A standard way $[7,14,24]$ to localize it consists in using rational approximations. Here, we use the approach previously introduced in [14] in the framework of OSRC methods. We introduce the rational Padé approximation of order $N_{p}$ of the

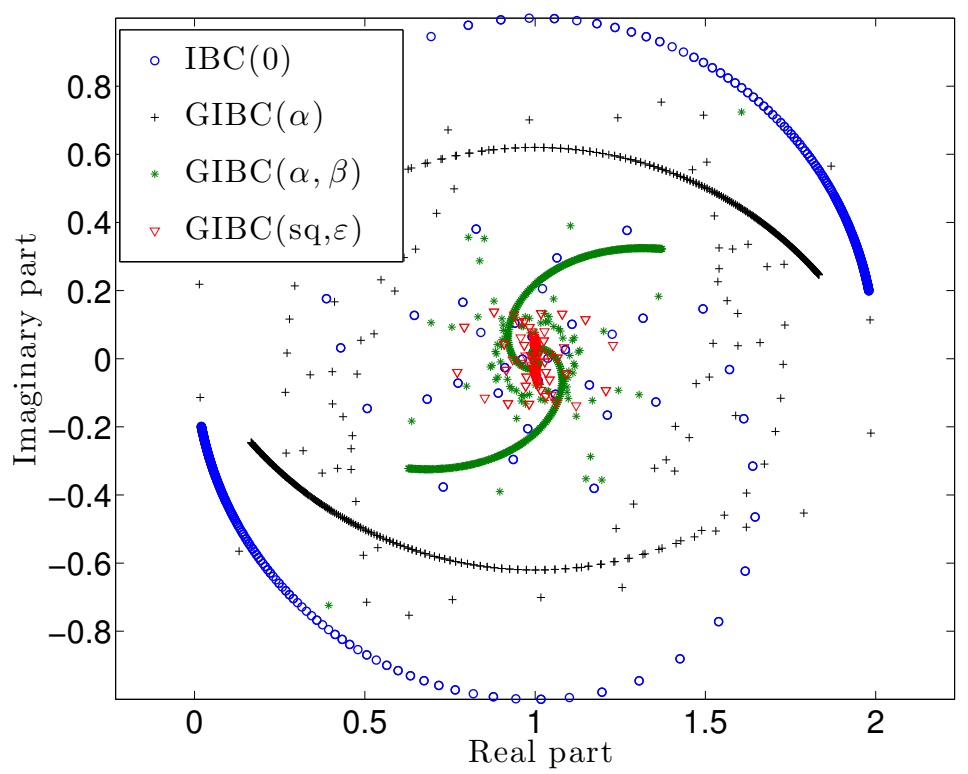

Figure 3: Eigenvalues distribution of the operator $(\mathcal{I}-\mathcal{A})$ for the different GIBCs. 
square-root function [24] with a rotation of the branch-cut

$$
(1+z)^{1 / 2} \approx e^{\imath \frac{\theta_{p}}{2}} R_{N_{p}}\left((1+z) e^{-\imath \theta_{p}}-1\right)=C_{0}+\sum_{\ell=1}^{N_{p}} \frac{A_{\ell} z}{1+B_{\ell} z}=R_{0}-\sum_{\ell=1}^{N_{p}} \frac{A_{\ell}}{B_{\ell}\left(1+B_{\ell} z\right)},
$$

where $R_{N_{p}}$ is the standard real-valued Padé approximation of order $N_{p}$

$$
(1+z)^{1 / 2} \approx R_{N_{p}}(z)=1+\sum_{\ell=1}^{N_{p}} \frac{a_{\ell} z}{1+b_{\ell} z}
$$

and

$$
a_{\ell}=\frac{2}{2 N_{p}+1} \sin ^{2}\left(\frac{\ell \pi}{2 N_{p}+1}\right), \quad b_{\ell}=\cos ^{2}\left(\frac{\ell \pi}{2 N_{p}+1}\right) .
$$

The angle of rotation $\theta_{p}$ is a free parameter that is fixed for the numerical simulations and

$$
\begin{aligned}
C_{0} & =e^{\imath \frac{\theta_{p}}{2}} R_{N_{p}}\left(e^{-\imath \theta_{p}}-1\right), & A_{\ell} & =\frac{e^{-\imath \frac{\theta_{p}}{2}} a_{\ell}}{\left(1+b_{\ell}\left(e^{-\imath \theta_{p}}-1\right)\right)^{2}}, \\
B_{\ell} & =\frac{e^{-\imath \theta_{p}} b_{\ell}}{1+b_{\ell}\left(e^{-\imath \theta_{p}}-1\right)}, & R_{0} & =C_{0}+\sum_{\ell=1}^{N_{p}} \frac{A_{\ell}}{B_{\ell}} .
\end{aligned}
$$

If one formally considers that $z=\mathscr{T}$, then $\boldsymbol{\Lambda}_{1, \varepsilon}$ can be approximated by

$$
\mathbf{\Lambda}_{1, \varepsilon}=(\mathbf{I}+\mathscr{T})^{1 / 2} \approx \tilde{\mathbf{\Lambda}}_{1, \varepsilon}:=\left(R_{0}-\sum_{\ell=1}^{N_{p}} \frac{A_{\ell}}{B_{\ell}}\left(\mathbf{I}+B_{\ell} \mathscr{T}\right)^{-1}\right) .
$$

Now, if we use this approximation, the equation (41) and we introduce $N_{p}$ coupled auxiliary vector fields $\left\{\boldsymbol{\phi}^{\ell}\right\}_{\ell=1, \ldots, N_{p}}$, we obtain a local and approximate computation of $\boldsymbol{\Lambda}_{1, \varepsilon} \mathbf{M}$ through $\tilde{\boldsymbol{\Lambda}}_{1, \varepsilon} \mathbf{M}$, which is realized through the system

$$
\left\{\begin{array}{l}
\tilde{\mathbf{\Lambda}}_{1, \varepsilon} \mathbf{M}=R_{0} \mathbf{M}-\sum_{\ell=1}^{N_{p}} \frac{A_{\ell}}{B_{\ell}} \boldsymbol{\phi}^{\ell}, \quad \text { on } \Gamma, \\
\mathbf{M}-\left(\mathbf{I}+B_{\ell}\left(\nabla_{\Gamma} \frac{1}{k_{\varepsilon}^{2}} \operatorname{div}_{\Gamma}-\operatorname{curl}_{\Gamma} \frac{1}{k_{\varepsilon}^{2}} \operatorname{curl}_{\Gamma}\right)\right) \boldsymbol{\phi}^{\ell}=\mathbf{0}, \ell=1, \ldots, N_{p}, \quad \text { on } \Gamma,
\end{array}\right.
$$

setting $i k \mathbf{M}=\gamma^{t}(\mathbf{c u r l} \mathbf{E})$. The way we use this representation in a DDM is explained in Section 6 .

Since we use an approximation of the square-root GIBC, this clearly modifies the spectrum of the operator $(\mathcal{I}-\mathcal{A})$ that we wish to solve. To illustrate this point, we report on Figure 4 the spectrum of $(\mathcal{I}-\mathcal{A})$ when using $\operatorname{GIBC}(\mathrm{sq}, \varepsilon)$ and $\operatorname{GIBC}\left(N_{p}, \theta_{p}, \varepsilon\right)\left(\right.$ denoted by $\left(\mathcal{I}-\mathcal{A}^{N_{p}, \theta_{p}}\right)$ ) related to the Padé approximation, and for the numerical situation described in section 4 . In [14], we have numerically shown that $\theta_{p}=\pi / 2$ leads to the most accurate $\operatorname{GIBC}\left(N_{p}, \theta_{p}, \varepsilon\right)$ in the framework of OSRC methods. For the DDM, some numerical simulations show that $\theta_{p}=\pi / 2$ also leads to the best convergence results when using FEMs. As we have seen before for the square-root operator, the eigenvalues of $\mathbb{A}_{m}$ are such that $\lambda_{m, 1}=\lambda_{m, 3}$ and $\lambda_{m, 2}=\lambda_{m, 4}$. This is no longer true when using a rational approximation. Indeed, we can see that we have four distinct eigenvalues $\lambda_{m, 1}^{N_{p}, \theta_{p}}=-\lambda_{m, 2}^{N_{p}, \theta_{p}}$ and $\lambda_{m, 3}^{N_{p}, \theta_{p}}=-\lambda_{m, 4}^{N_{p}, \theta_{p}}$ for the associated iteration operator $\mathbb{A}_{m}^{N_{p}, \theta_{p}}$, but with $\lambda_{m, 1}^{N_{p}, \theta_{p}} \neq \lambda_{m, 3}^{N_{p}, \theta_{p}}$. Furthermore, for $N_{p} \geq 4$, we observe here that the spectra of the two operators $(\mathcal{I}-\mathcal{A})$ and $\left(\mathcal{I}-\mathcal{A}^{N_{p}, \theta_{p}}\right)$ are very close, with a large clustering around $1+\frac{\imath \varepsilon}{2 k+\imath \varepsilon}$.

Since we perturbed the eigenvalues when using rational approximants, the convergence of the Krylov subspace solvers is impacted. We report on Figure 5 the GMRES residual history with respect to \#iter when solving the DDM with spherical decomposition for the same situation as in Section 4.1 , based on $\left(\mathcal{I}-\mathcal{A}^{N_{p}, \theta_{p}}\right)$. We clearly see how $N_{p}$ affects the convergence rate, $N_{p}=8$ being optimal in this situation, even if $N_{p}=4$ already leads to a very good convergence. 


\section{Finite element formulation of the DDM with GIBC}

Let us now explain the implementation of the domain decomposition algorithm when using the Padé-type transmission boundary conditions in a finite element context. We recall that the iterative method consists in solving the subproblems (3) and then computing the transmitted vector fields (4). For the sake of conciseness, we describe the situation where the problem is set in a bounded subdomain that we call $\Omega$ in this Section. It has no exterior nor interface connected to the scattering object. Therefore, we have: $\Gamma_{i}=\emptyset, \Gamma_{i}^{\infty}$ and $\Gamma:=\partial \Omega$ is an artificial interface. Extending the formulation to the other situations is straightforward. For the sake of clarity, we abbreviate the notations $\mathbf{E}_{i}^{p+1}, \mathbf{g}_{i j}^{p}, \mathbf{g}_{j i}^{p+1}$ by $\mathbf{E}, \mathbf{g}^{\text {in }}, \mathbf{g}^{\text {out }}$, respectively, used in systems (3) and (4). Then, we first
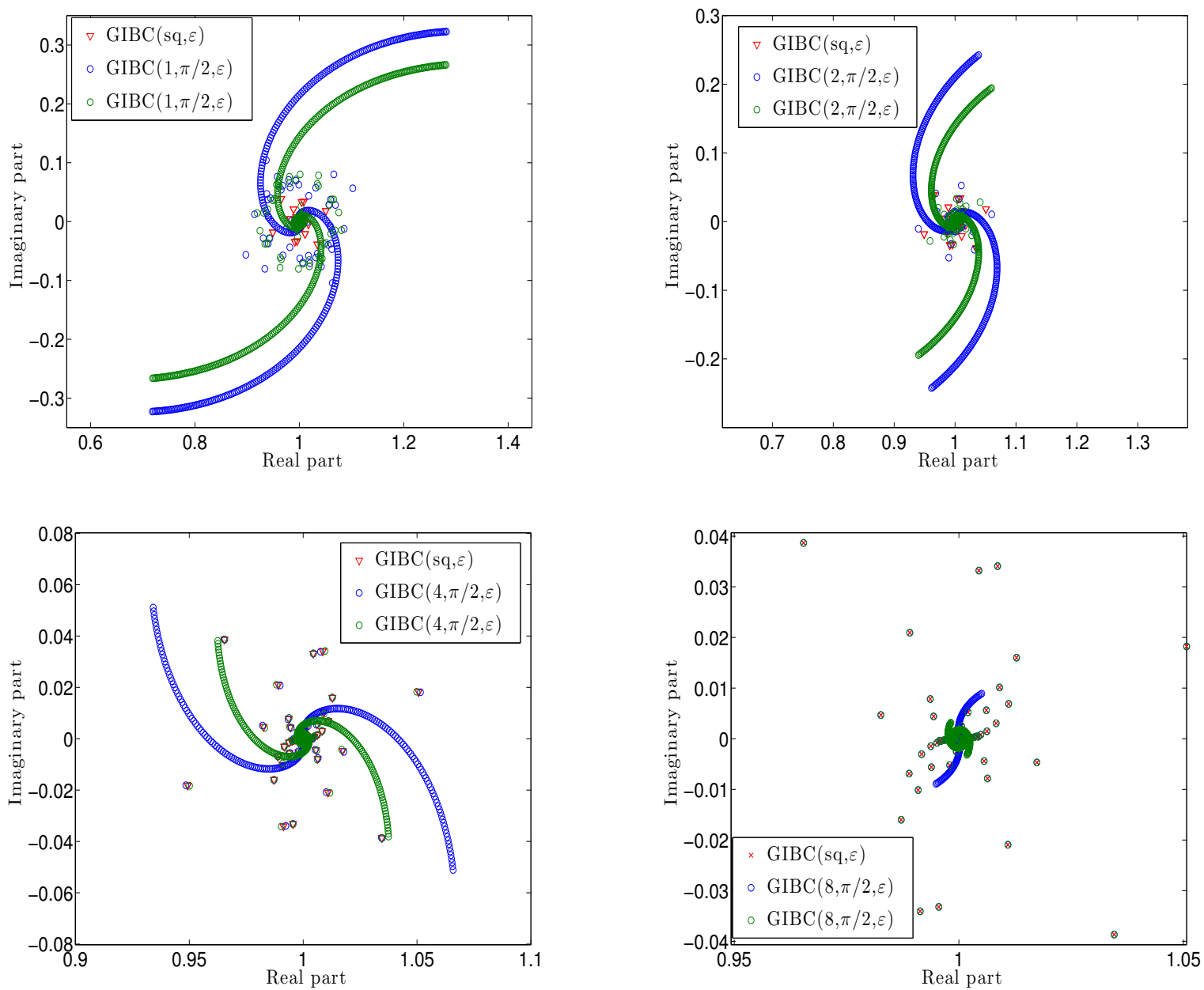

Figure 4: Distribution of the eigenvalues of the operators $(\mathcal{I}-\mathcal{A})$ and $\left(\mathcal{I}-\mathcal{A}^{N_{p}, \theta_{p}}\right)$ in the complex plane. 


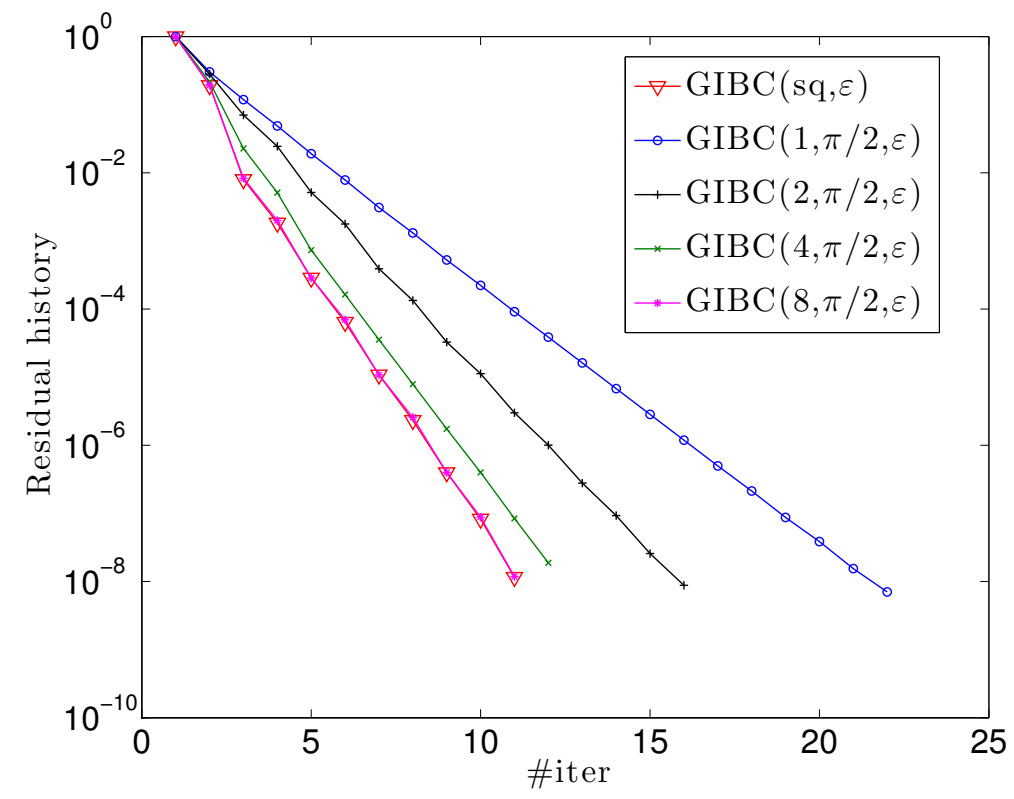

Figure 5: Residual history of GMRES vs. \#iter for the square-root and Padé-type GIBCs.

have

$$
\left\{\begin{array}{l}
\operatorname{curl} \operatorname{curl} \mathbf{E}-k^{2} \mathbf{E}=0, \quad \text { in } \Omega, \\
\frac{1}{R_{0}} \mathbf{\Lambda}_{2, \varepsilon}\left(\gamma^{T}(\mathbf{E})\right)+\left(\mathbf{M}+\sum_{\ell=1}^{N_{p}} \alpha_{\ell} \phi^{\ell}\right):= \\
\frac{1}{R_{0}}\left(\mathbf{I}-\operatorname{curl}_{\Gamma} \frac{1}{k_{\varepsilon}^{2}} \operatorname{curl}_{\Gamma}\right)\left(\gamma^{T}(\mathbf{E})\right)+\left(\mathbf{M}+\sum_{\ell=1}^{N_{p}} \alpha_{\ell} \boldsymbol{\phi}^{\ell}\right)=\frac{1}{R_{0}} \mathbf{g}^{\text {in }}, \quad \text { on } \Gamma, \\
\mathbf{M}-\left(\mathbf{I}+B_{\ell}\left(\nabla_{\Gamma} \frac{1}{k_{\varepsilon}^{2}} \operatorname{div}_{\Gamma}-\operatorname{curl}_{\Gamma} \frac{1}{k_{\varepsilon}^{2}} \operatorname{curl}_{\Gamma}\right)\right) \boldsymbol{\phi}^{\ell}=\mathbf{0}, \ell=1, \ldots, N_{p}, \quad \text { on } \Gamma,
\end{array}\right.
$$

where $i k \mathbf{M}=\gamma^{t}(\mathbf{c u r l} \mathbf{E})$ and $\alpha_{\ell}=-A_{\ell} /\left(R_{0} B_{\ell}\right)$. The updating equation is (from equation (12))

$$
\mathbf{g}^{\text {out }}=-\mathbf{g}^{\text {in }}+2 \boldsymbol{\Lambda}_{2, \varepsilon}\left(\gamma^{T}(\mathbf{E})\right), \quad \text { on } \Sigma .
$$

Let us consider now that $\Omega$ and $\Gamma$ are respectively meshed by using $N_{t}^{\Omega}$ tetrahedra and $N_{t}^{\Gamma}$ surface triangles, respectively, resulting in the discrete domains: $\Omega_{h}=\cup_{q=1}^{N_{t}^{\Omega}} \mathcal{T}^{q}$ and $\Gamma_{h}:=\cup_{q=1}^{N_{t}^{\Gamma}} T^{q}$. Let us introduce: $\boldsymbol{\Phi}_{h}:=\left(\phi_{h}^{\ell}\right)_{\ell=1, \ldots, N_{p}}$ and $\boldsymbol{\rho}_{h}:=\left(\rho_{h}^{\ell}\right)_{\ell=1, \ldots, N_{p}}$. For solving system (47), we use the following symmetrical weak formulation: find $\left(\mathbf{E}_{h}, \mathbf{M}_{h}, \boldsymbol{\Phi}_{h}, \boldsymbol{\rho}_{h}\right) \in V_{h}^{\Omega} \times V_{h}^{\Gamma} \times V_{h}^{p} \times Z_{h}^{p}$ such that 


$$
\left\{\begin{array}{c}
\int_{\Omega_{h}}\left(\operatorname{curl} \mathbf{E}_{h} \cdot \mathbf{c u r l} \mathbf{W}_{h}-k^{2} \mathbf{E}_{h} \cdot \mathbf{W}_{h}\right) d \Omega_{h}+\imath k \int_{\Gamma_{h}} \mathbf{M}_{h} \cdot \mathbf{W}_{h} d \Gamma_{h}=0 \\
\frac{1}{R_{0}} \int_{\Gamma_{h}}\left(\gamma^{T}\left(\mathbf{E}_{h}\right) \cdot \mathbf{v}_{h}-\frac{1}{k_{\varepsilon, h}^{2}} \operatorname{curl}_{\Gamma_{h}} \gamma^{T}\left(\mathbf{E}_{h}\right) \cdot \operatorname{curl}_{\Gamma_{h}} \mathbf{v}_{h}\right) d \Gamma_{h}+\int_{\Gamma_{h}} \mathbf{M}_{h} \cdot \mathbf{v}_{h} d \Gamma_{h} \\
\quad+\sum_{\ell=1}^{N_{p}} \alpha_{\ell} \int_{\Gamma_{h}} \phi_{h}^{\ell} \cdot \mathbf{v}_{h} d \Gamma_{h}=\frac{1}{R_{0}} \int_{\Gamma_{h}} \mathbf{g}_{h}^{\text {in }} \cdot \mathbf{v}_{h} d \Gamma_{h}, \\
\int_{\Gamma_{h}} \mathbf{M}_{h} \cdot \mathbf{w}_{h}^{\ell} d \Gamma_{h}-\int_{\Gamma_{h}}\left(\phi_{h}^{\ell} \cdot \mathbf{w}_{h}^{\ell}-\frac{B_{\ell}}{k_{\varepsilon, h}^{2}} \operatorname{curl}_{\Gamma_{h}} \phi_{h}^{\ell} \cdot \operatorname{curl}_{\Gamma_{h}} \mathbf{w}_{h}^{\ell}\right) d \Gamma_{h} \\
\quad-B_{\ell} \int_{\Gamma_{h}} \nabla_{\Gamma_{h}} \rho_{h}^{\ell} \cdot \mathbf{w}_{h}^{\ell} d \Gamma_{h}=0, \quad \ell=1, \ldots, N_{p}, \\
\int_{\Gamma_{h}}\left(k_{\varepsilon, h}^{2} \rho_{h}^{\ell} z_{h}^{\ell}+\phi_{h}^{\ell} \cdot \nabla_{\Gamma_{h}} z_{h}^{\ell}\right) d \Gamma_{h}=0, \quad \ell=1, \ldots, N_{p},
\end{array}\right.
$$

for any test-functions $\mathbf{W}_{h} \in V_{h}^{\Omega}, \mathbf{v}_{h}$ and $\mathbf{w}_{h}^{\ell}$ in $V_{h}^{\Gamma}$, and $z_{h}^{\ell}$ in $Z_{h}$, for $\ell=1, \ldots, N_{p}$. After solving (49), the boundary data is updated through the relation

$$
\mathbf{g}_{h}^{\text {out }}=\mathbf{g}_{h}^{\text {in }}-2 R_{0} \mathbf{M}_{h}-2 R_{0} \sum_{\ell=1}^{N_{p}} \alpha_{\ell} \phi_{h}^{\ell} .
$$

The approximation spaces $V_{h}^{\Omega}$ and $V_{h}^{\Gamma}$ are the usual volume and surface Nédélec's space of lowest order edge finite element [25], respectively. If $N_{e}^{\Omega}$ (resp. $N_{e}^{\Gamma}$ ) is the number of edges of $\Omega_{h}$ (resp. $\Gamma_{h}$ ), we have: $\operatorname{dim}\left(V_{h}^{\Omega}\right)=N_{e}^{\Omega}$ (resp. $\operatorname{dim}\left(V_{h}^{\Gamma}\right)=N_{e}^{\Gamma}$ ). The space $V_{h}^{p}$, which is defined by: $V_{h}^{p}:=\times_{\ell=1}^{N_{p}} V_{h}$, is of dimension $N_{e}^{p}:=N_{p} N_{e}^{\Gamma}$. The approximation space $Z_{h}$ is the nodal finite element space

$$
Z_{h}=\left\{z_{h} \in \mathbf{H}^{-1 / 2}\left(\Gamma_{h}\right) \mid z_{h \mid T^{q}} \in \mathbb{P}_{1}\left(T^{q}\right), \forall q=1, \ldots, N_{t}^{\Gamma}\right\},
$$

where $\mathbb{P}_{1}$ is the space of complex-valued linear functions. In particular, we have: $\operatorname{dim}\left(Z_{h}\right)=N_{v}^{\Gamma}$, where $N_{v}^{\Gamma}$ is the number of vertices of the triangulation of $\Gamma_{h}$. We also define: $Z_{h}^{p}:=\times_{j=1}^{N_{p}} Z_{h}$, with dimension $N_{v}^{p}:=N_{p} N_{v}$. The function $k_{\varepsilon, h}$ is the linear interpolation of $k_{\varepsilon}$ over $\Gamma_{h}$. Let us now introduce $\left(\mathbf{R}_{1}, \ldots, \mathbf{R}_{N_{e}^{\Omega}}\right),\left(\mathbf{r}_{1}, \ldots, \mathbf{r}_{N_{e}^{\Gamma}}\right)$ and $\left(\ell_{1}, \ldots, \ell_{N_{v}^{\Gamma}}\right)$ as the basis of $V_{h}^{\Omega}, V_{h}^{\Gamma}$ and $Z_{h}$, respectively. Then, one gets for $\mathbf{W}_{h} \in V_{h}^{\Omega}, \mathbf{v}_{h} \in V_{h}^{\Gamma}$ and $z_{h} \in Z_{h}$

$$
\mathbf{W}_{h}=\sum_{q=1}^{N_{e}^{\Omega}} W_{q} \mathbf{R}_{q}, \quad \mathbf{v}_{h}=\sum_{q=1}^{N_{e}^{\Gamma}} v_{q} \mathbf{r}_{q} \quad \text { and } \quad z_{h}=\sum_{m=1}^{N_{v}^{\Gamma}} u_{m} \ell_{m} .
$$

Let us now define the elementary integrals

$$
\begin{cases}\mathbb{A}_{u v}^{\Omega}=\int_{\Omega_{h}}\left(\operatorname{curl} \mathbf{R}_{u} \cdot \operatorname{curl} \mathbf{R}_{v}-k^{2} \mathbf{R}_{u} \cdot \mathbf{R}_{v}\right) d \Omega_{h}, \\ \mathbb{A}_{p q}=\int_{\Gamma_{h}}^{\mathbf{r}_{p} \cdot \mathbf{r}_{q} d \Gamma_{h},} \quad \mathbb{N}_{p q}=\int_{\Gamma_{h}} \frac{1}{k_{\varepsilon, h}^{2}} \operatorname{curl}_{\Gamma_{h}} \mathbf{r}_{p} \cdot \operatorname{curl}_{\Gamma} \mathbf{r}_{q} d \Gamma_{h}, \\ \mathbb{K}_{m n}=\int_{\Gamma_{h}} k_{\varepsilon, h}^{2} \ell_{m} \ell_{n} d \Gamma_{h}, & \mathbb{L}_{m q}=\int_{\Gamma_{h}} \nabla_{\Gamma_{h}} \ell_{m} \cdot \mathbf{r}_{q} d \Gamma_{h},\end{cases}
$$

with $1 \leq u, v \leq N_{e}^{\Omega}, 1 \leq p, q \leq N_{e}^{\Gamma}$ and $1 \leq m, n \leq N_{v}^{\Gamma}$. The associated matrix $\mathbb{A}^{\Omega} \in \mathcal{M}_{N_{e}^{\Omega}, N_{e}^{\Omega}}(\mathbb{C})$, $\mathbb{A}$ and $\mathbb{N}$ are in $\mathcal{M}_{N_{e}^{\Gamma}, N_{e}^{\Gamma}}(\mathbb{C}), \mathbb{K} \in \mathcal{M}_{N_{v}^{\Gamma}, N_{v}^{\Gamma}}(\mathbb{C})$ and $\mathbb{L} \in \mathcal{M}_{N_{e}^{\Gamma}, N_{v}^{\Gamma}}(\mathbb{C})$. Under these notations, the system (49) can be written as the system of coupled equations

$$
\mathbb{M} \mathbf{U}_{h}=\mathbf{F}_{h},
$$


where we define

$$
\mathbb{M}=\left[\begin{array}{cccc}
\mathbb{A}^{\Omega} & \mathbb{D} & \mathbf{0} & \mathbf{0} \\
\mathbb{E} & \mathbb{A} & \mathbb{A}_{\boldsymbol{\alpha}} & \mathbf{0} \\
\mathbf{0} & \mathbb{A}_{\boldsymbol{\alpha}}^{T} & \mathbb{A}_{\boldsymbol{\alpha}, \mathbf{B}} & \mathbb{A}_{\boldsymbol{\alpha}, \mathbf{B}}^{\mathbb{L}} \\
\mathbf{0} & \mathbf{0} & \mathbb{A}_{\boldsymbol{\alpha}, \mathbf{B}}^{\mathbb{L},} & \mathbb{A}_{\boldsymbol{\alpha}, \mathbf{B}}^{\mathbb{K}}
\end{array}\right], \mathbf{U}_{h}=\left[\begin{array}{c}
\mathbf{E}_{h} \\
\mathbf{M}_{h} \\
\mathbf{\Phi}_{h} \\
\boldsymbol{\rho}_{h}
\end{array}\right], \mathbf{F}_{h}=\left[\begin{array}{c}
\mathbf{0} \\
\mathbf{F}_{2, h} \\
\mathbf{0} \\
\mathbf{0}
\end{array}\right]
$$

with

$$
\begin{aligned}
& \mathbb{D}:=\imath k \mathbb{A} \in \mathcal{M}_{N_{e}^{\Omega}, N_{e}^{\Gamma}}(\mathbb{C}), \\
& \mathbb{E}:=\frac{(\mathbb{A}+\mathbb{N})}{R_{0}} \in \mathcal{M}_{N_{e}^{\Gamma}, N_{e}^{\Omega}}(\mathbb{C}), \\
& \mathbb{A}_{\boldsymbol{\alpha}}:=\left(\left(\alpha_{\ell} \mathbb{A}\right)_{\ell=1, \ldots, N_{p}}\right) \in \mathcal{M}_{N_{e}^{\Gamma}, N_{e}^{p}}(\mathbb{C}), \\
& \mathbb{A}_{\boldsymbol{\alpha}, \mathbf{B}}:=\operatorname{diag}\left(\alpha_{\ell}\left(B_{\ell} \mathbb{N}-\mathbb{A}\right)_{\ell=1, \ldots, N_{p}}\right) \in \mathcal{M}_{N_{e}^{p}, N_{e}^{p}}(\mathbb{C}), \\
& \mathbb{A}_{\boldsymbol{\alpha}, \mathbf{B}}^{\mathbb{L}}:=\operatorname{diag}\left(\left(-\alpha_{\ell} B_{\ell} \mathbb{L}\right)_{\ell=1, \ldots, N_{p}}\right) \in \mathcal{M}_{N_{e}^{p}, N_{v}^{p}}(\mathbb{C}), \\
& \mathbb{A}_{\boldsymbol{\alpha}, \mathbf{B}}^{\mathbb{K}}:=\operatorname{diag}\left(\left(-\alpha_{\ell} B_{\ell} \mathbb{K}\right)_{\ell=1, \ldots, N_{p}}\right) \in \mathcal{M}_{N_{v}^{p}, N_{v}^{p}}(\mathbb{C}), \\
& \mathbf{F}_{2, h}:=\frac{\mathbb{A}}{R_{0}} \mathbf{g}_{h}^{\text {in }} \in \mathbb{C}^{N_{e}^{\Gamma}} .
\end{aligned}
$$

By $A^{T}$, we designate the transposed of a given complex-valued matrix $A$. The sparse complexvalued matrix $\mathbb{M}$ of size $n_{p} \times n_{p}$ (with $n_{p}:=N_{e}^{\Omega}+N_{e}^{\Gamma}+N_{e}^{p}+N_{v}^{p}$ ) that defines the linear system (52) is non-definite positive. In addition, the vector fields $\mathbf{U}_{h}$ and $\mathbf{F}_{h}$ are in $\mathbb{C}^{n_{1, p}}$. Finally, the boundary data are updated by using (50).

\section{Numerical results}

In this section, we present some numerical examples concerning the performance of the DDMs introduced in this paper and compare the various transmission boundary conditions. Three scatterers are considered: a circular cylinder, a sphere and a Falcon jet. All the numerical tests were performed using the open source software package GetDDM, which combines the open source mesh generator Gmsh [19] and the finite solver GetDP [13, 18], and is available online for further testing at the address http://onelab.info/wiki/GetDDM together the input data files for the first two examples.

We first analyze in detail the case of a circular cylinder of radius $R=1$ under TE $\left(\mathbf{E}^{\text {inc }}=\right.$ $\mathbf{e}_{z} e^{-i k x}$; Figure 6$)$ and TM $\left(\mathbf{E}^{\text {inc }}=\mathbf{e}_{y} e^{-i k x}\right.$; Figure 7) plane wave incidence. The outer circular boundary delimitating the computational domain is set to $\Gamma^{\infty}=\mathcal{C}_{5}$, for a Silver-Müller absorbing boundary condition. The domain of computation is cut into $N_{\text {dom }}=5$ concentric subdomains (see Figure 8) in such a way that the local number of degrees of freedom (involved in the FEM) for each subdomain is well-balanced to optimize the domain decomposition algorithm. We report on Figure 6 the number of iterations \#iter of the GMRES without restart (with relative tolerance equal to $10^{-6}$ ) for the DDM vs. the wavenumber $k$. Two densities of discretization points per wavelength $n_{\lambda}$ are presented: $n_{\lambda}=10$ (top) and $n_{\lambda}=20$ (bottom). In each case, we compare various transmission boundary conditions: $\operatorname{IBC}(0), \operatorname{GIBC}(\alpha, \beta)$ and $\operatorname{GIBC}\left(N_{p}, \pi / 2, \varepsilon\right)$, for $N_{p}=1,2,4,8$ and $\varepsilon=0.39 k^{1 / 3} R_{\Sigma_{i j}}^{-2 / 3}$. For the TE polarization case, we observe that the number of iterations for $\operatorname{IBC}(0), \operatorname{GIBC}(\alpha, \beta)$ and $\operatorname{GIBC}(1, \pi / 2, \varepsilon)$ depends on $k$ while this does not seem to be the case for $\operatorname{GIBC}\left(N_{p}, \pi / 2, \varepsilon\right)$, with $N_{p}=2,4,8$. For example, for $k=30$ and $n_{\lambda}=20, \operatorname{IBC}(0)$ requires 56 iterations, $\operatorname{GIBC}(\alpha, \beta)$ needs 35 iterations while $\operatorname{GIBC}(4, \pi / 2, \varepsilon)$ converges in 21 iterations. For the TM polarization, we observe on Figure 7 the same behavior even if $\operatorname{GIBC}(\alpha, \beta)$ seems to depend less on $k$ than for the TE case. Also, in all cases the convergence behavior of the RawatLee condition $\operatorname{GIBC}(\alpha, \beta)$ is fairly similar to the convergence of the lowest order Padé-localized condition $\operatorname{GIBC}(1, \pi / 2, \varepsilon)$, which is consistent with the remark made at the end of Section 3.2. 
Finally, we report on Figure 8 the modulus of the reconstructed electromagnetic scattered field for $k=10$, for the TE polarization case.

The second test case concerns the scattering by the unit sphere. The fictitious boundary $\Gamma^{\infty}$ is the sphere of radius 2. We report on Figure 9 the residual history of the GMRES without restart for two subdomains, for $k=20$ and $n_{\lambda}=10$. We can see that $\operatorname{IBC}(0)$ requires many iterations. Using $\operatorname{GIBC}(\alpha, \beta)$ needs $50 \%$ more iterations than $\operatorname{GIBC}(4, \pi / 2, \varepsilon)$ to converge with a relative residual equal to $10^{-6}$.

The third example presents the scattering problem of an electromagnetic wave by a Falcon jet (see Figure 10). The computation involves $N_{\text {dom }}=4$ subdomains (Figure 10, top). Figure 10 (bottom) reports the electromagnetic field in a vertical slice computed by the domain decomposition method. We observe on Figure 11 that the converge of the GMRES (for a residual equal to $10^{-5}$ ) is obtained for $\operatorname{IBC}(0)$ in about 100 iterations, for $\operatorname{GIBC}(\alpha, \beta)$ in 30 iterations and in 18 iterations for $\operatorname{GIBC}(2, \pi / 2, \varepsilon)$.

\section{Conclusion}

In this paper we have proposed and analyzed a high-order optimized Schwarz domain decomposition method for the time harmonic Maxwell equations, which exhibits quasi-optimal convergence properties. The algorithm is based on high-order rational approximations of the Magnetic-to-Electric operator on the transmitting interfaces between the subdomains. The resulting code and examples are freely available online for further testing.

As seen in this paper for electromagnetics, and in [7] for acoustics, once a suitable high-order transmitting operator has been built, the optimized Schwarz domain decomposition method exhibits quasi-optimal convergence. Future work could thus deal with the application of the same methodology to elastodynamics, based on the recent work by Darbas et al. [8].

\section{Acknowledgement}

This work was supported in part by the Belgian Science Policy (PAI grant P7/02), the Walloon Region (WIST3 grants ONELAB and ALIZEES), the French ANR (grant MicroWave NT09 460489 "Programme Blanc") and the "EADS Foundation" (High-BRID project, grant 089-1009-1006).

Computational resources have been provided by CÉCI, funded by F.R.S.-FNRS (Fonds de la Recherche Scientifique) under grant $\mathrm{n}^{\circ}$ 2.5020.11, and the Tier-1 supercomputer of the Fédération Wallonie-Bruxelles, funded by the Walloon Region under grant $n^{\circ} 1117545$.

\section{References}

[1] A. Alonso-Rodriguez and L. Gerardo-Giorda. New nonoverlapping domain decomposition methods for the harmonic Maxwell system. SIAM J. Sci. Comput., 28(1):102-122, 2006.

[2] M. Abramowitz and I. Stegun. Handbook of Mathematical Functions: With Formulas, Graphs, and Mathematical Tables. Applied mathematics series. Dover Publications, 1964.

[3] H. Ammari. Scattering of waves by thin periodic layers at high frequencies using the on-surface radiation condition method. IMA J. Appl. Math., 60:199-215, 1997.

[4] X. Antoine. Fast approximate computation of a time-harmonic scattered field using the onsurface radiation condition method. IMA J. Appl. Math., 66(1):83-110, 2001. 
[5] X. Antoine. Advances in the on-surface radiation condition method: Theory, numerics and applications. In Computational Methods for Acoustics Problems, pages 169-194. Saxe-Coburg Publications, 2008.

[6] A. Bayliss and E. Turkel. Radiation boundary conditions for wave-like equations. Comm. Pure Appl. Math., 33(6):707-725, 1980.

[7] Y. Boubendir, X. Antoine, and C. Geuzaine. A quasi-optimal non-overlapping domain decomposition algorithm for the Helmholtz equation. Journal of Computational Physics, 2(231):262$280,2012$.

[8] M. Darbas and F. Le Louër. Well-conditioned boundary integral formulations for highfrequency elastic scattering problems in three dimensions. Mathematical Methods in the Applied Sciences, 2014.

[9] B. Després. Décomposition de domaine et problème de Helmholtz. C.R. Acad. Sci. Paris, $1(6): 313-316,1990$

[10] B. Després, P. Joly, and J. E. Roberts. A domain decomposition method for the harmonic Maxwell equations. In Iterative methods in linear algebra (Brussels, 1991), pages 475-484, Amsterdam, 1992. North-Holland.

[11] V. Dolean, J. M. Gander, S. Lanteri, J.-F. Lee, and Z. Peng. Optimized Schwarz methods for curl-curl time-harmonic Maxwell's equations. In J. Erhel, M. J. Gander, L. Halpern, T. Sassi, and $O$. Widlund, editors, Proceedings of the 21st international domain decomposition conference. Springer LNCSE, 2013.

[12] V. Dolean, M. J. Gander, and L. Gerardo-Giorda. Optimized Schwarz methods for Maxwell's equations. SIAM J. Sci. Comput., 31(3):2193-2213, 2009.

[13] P. Dular, C. Geuzaine, F. Henrotte, and W. Legros. A general environment for the treatment of discrete problems and its application to the finite element method. IEEE Transactions on Magnetics, 34(5):3395-3398, 1998.

[14] M. El Bouajaji, X. Antoine, and C. Geuzaine. Approximate local magnetic-to-electric surface operators for time-harmonic Maxwell's equations. Journal of Computational Physics, to appear, 2015.

[15] M. El Bouajaji, V. Dolean, M. J. Gander, and S. Lanteri. Optimized Schwarz methods for the time-harmonic Maxwell equations with damping. SIAM J. Sci. Comput., 34(4):A2048-A2071, 2012 .

[16] O. G. Ernst and M. J. Gander. Why it is difficult to solve Helmholtz problems with classical iterative methods. In Numerical analysis of multiscale problems, pages 325-363. Springer, 2012 .

[17] M. J. Gander, F. Magoulès, and F. Nataf. Optimized Schwarz methods without overlap for the Helmholtz equation. SIAM J. Sci. Comput., 24(1):38-60 (electronic), 2002.

[18] C. Geuzaine. GetDP: a general finite-element solver for the de Rham complex. PAMM, 7(1):1010603-1010604, 2007.

[19] C. Geuzaine and J.-F. Remacle. Gmsh: A 3-d finite element mesh generator with built-in preand post-processing facilities. International Journal for Numerical Methods in Engineering, 79(11):1309-1331, 2009. 
[20] J. Jin. The Finite Element Method in Electromagnetics. John Wiley \& Sons, Incorporated, New York, second edition, 2002.

[21] G. A. Kriegsmann, A. Taflove, and K. R. Umashankar. A new formulation of electromagnetic wave scattering using an on-surface radiation boundary condition approach. IEEE Trans. Antennas and Propagation, 35(2):153-161, 1987.

[22] F. Magoulès, P. Iványi, and B. H. V. Topping. Non-overlapping Schwarz methods with optimized transmission conditions for the Helmholtz equation. Comput. Methods Appl. Mech. Engrg., 193(45-47):4797-4818, 2004.

[23] F. Magoulès and F.-X. Roux. Dirichlet-to-Neumann maps for domain decomposition methods: a unified approach. In Domain decomposition methods: theory and applications, volume 25 of GAKUTO Internat. Ser. Math. Sci. Appl., pages 123-145. Gakkōtosho, Tokyo, 2006.

[24] F. Milinazzo, C. Zala, and G. Brooke. Rational square-root approximations for parabolic equation algorithms. Journal of the Acoustical Society of America, 101(2):760-766, FEB 1997.

[25] P. Monk. Finite Element Methods for Maxwell's Equations. Numerical Mathematics and Scientific Computation. Oxford University, 2003.

[26] R. Murch. The on-surface radiation condition applied to three-dimensional convex objects. IEEE Trans. Antennas and Propagation, 41(5):651-658, 1993.

[27] J.-C. Nédélec. Acoustic and electromagnetic equations, volume 144 of Applied Mathematical Sciences. Springer-Verlag, New York, 2001. Integral representations for harmonic problems.

[28] Z. Peng and J.-F. Lee. A scalable nonoverlapping and nonconformal domain decomposition method for solving time-harmonic Maxwell equations in $\mathbb{R}^{3}$. SIAM J. Sci. Comput., 34(3):A1266-A1295, 2012.

[29] Z. Peng, V. Rawat, and J.-F. Lee. One way domain decomposition method with second order transmission conditions for solving electromagnetic wave problems. J. Comput. Phys., 229(4):1181-1197, 2010.

[30] V. Rawat and J.-F. Lee. Nonoverlapping domain decomposition with second order transmission condition for the time-harmonic Maxwell's equations. SIAM J. Scientific Computing, $32(6): 3584-3603,2010$.

[31] R. Roxburgh. Electromagnetic scattering from a right-circular cylinder using a surface radiation condition. IMA J. Appl. Math., 59:221-230, 1997.

[32] G. Watson. A Treatise on the Theory of Bessel Functions. Cambridge University Press, Cambridge, 1994. 

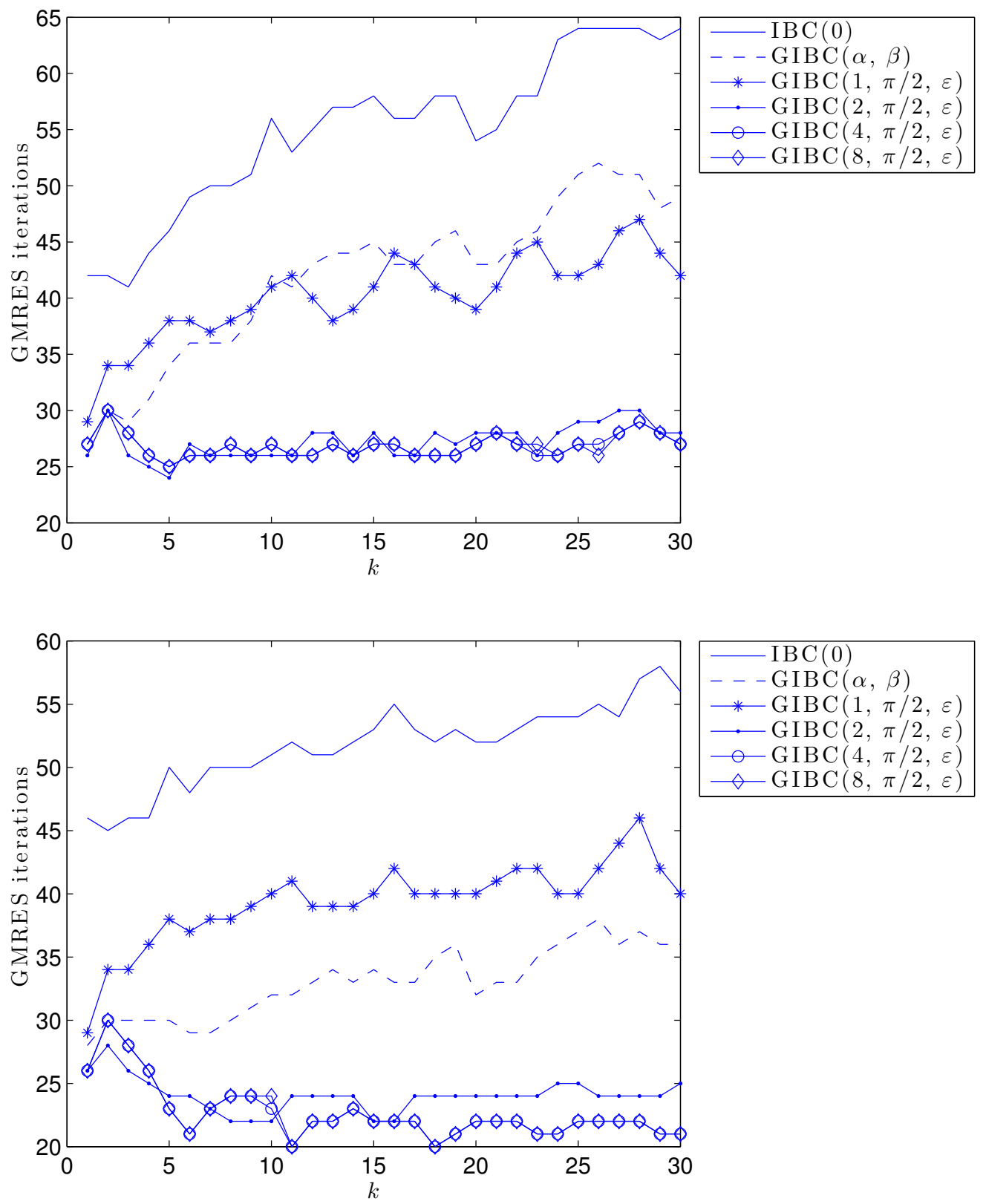

Figure 6: Circular cylinder under TE plane wave incidence: number of GMRES iterations \#iter vs. $k\left(N_{\text {dom }}=5, n_{\lambda}=10\right.$ (top) and $n_{\lambda}=20$ (bottom)). 

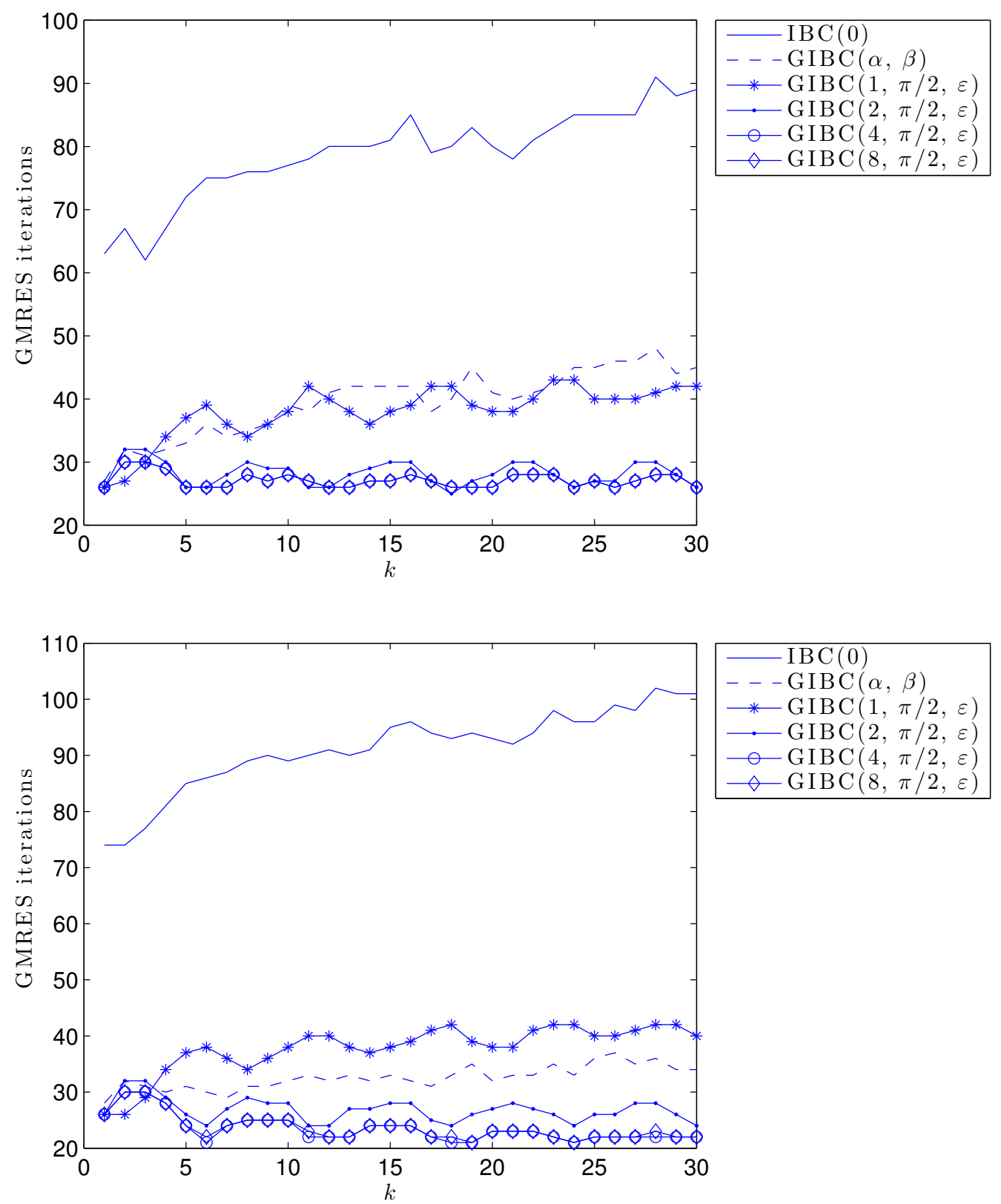

Figure 7: Circular cylinder under TM plane wave incidence: number of GMES iterations \#iter vs. $k\left(N_{\text {dom }}=5, n_{\lambda}=10\right.$ (top) and $n_{\lambda}=20$ (bottom) $)$. 


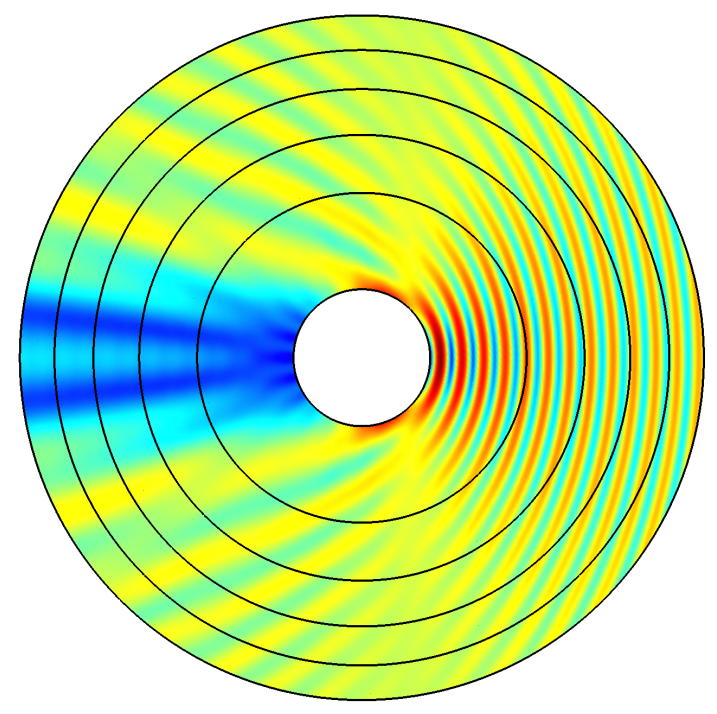

Figure 8: Circular cylinder under TE polarization: $\|\mathbf{E}\|\left(\right.$ for $N_{\text {dom }}=5$ and $\left.k=10\right)$.

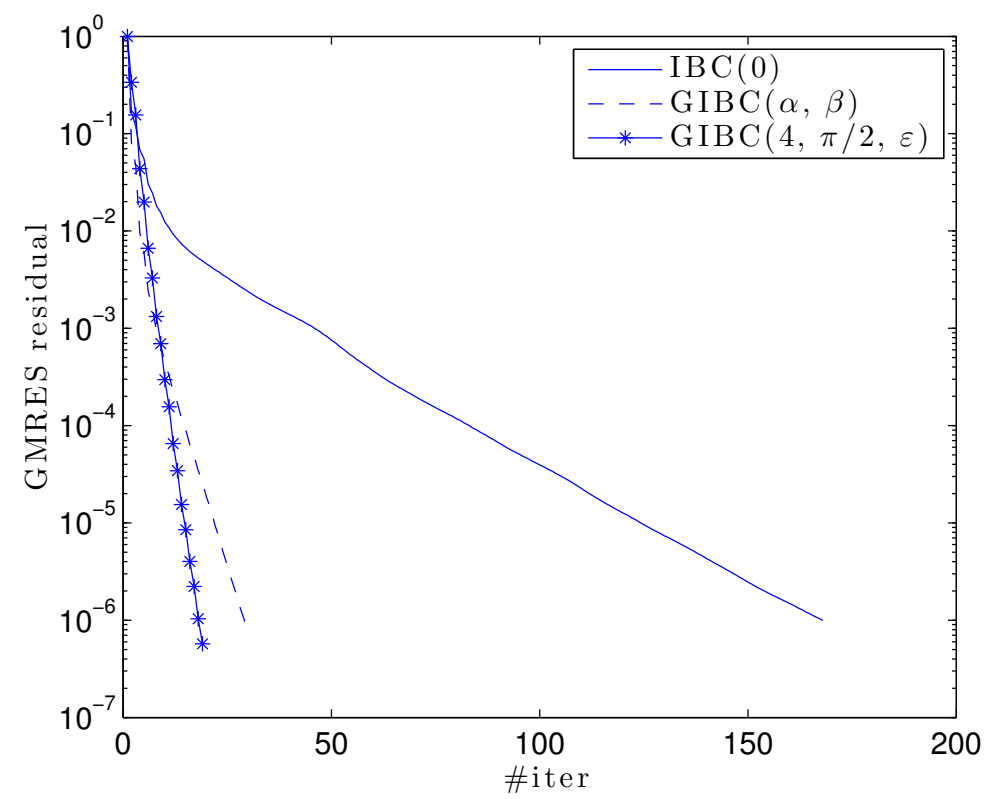

Figure 9: Unit sphere: residual history of the GMRES without restart $\left(N_{\mathrm{dom}}=2, k=20, n_{\lambda}=10\right)$. 

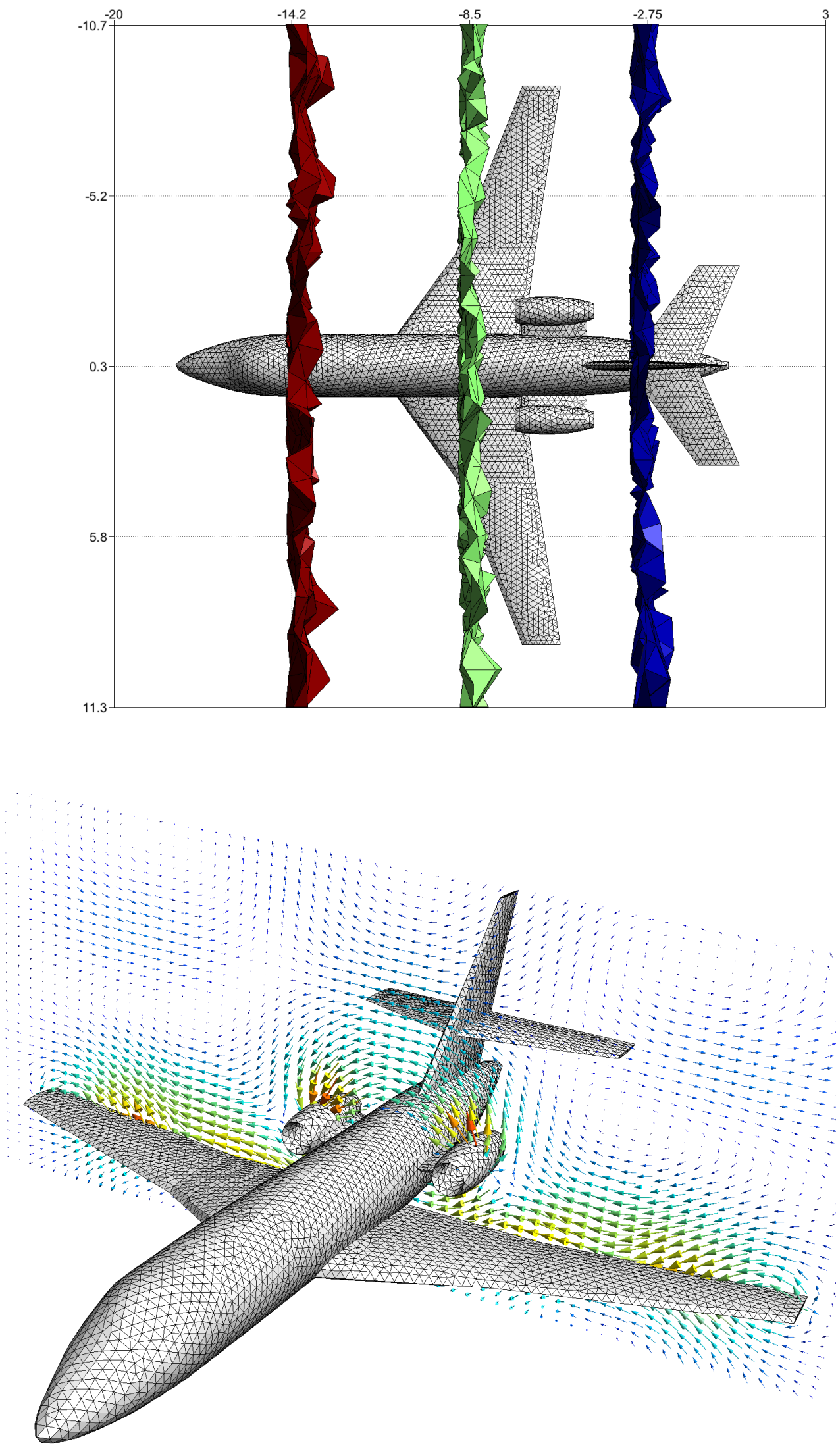

Figure 10: Electromagnetic scattering by a Falcon jet. 


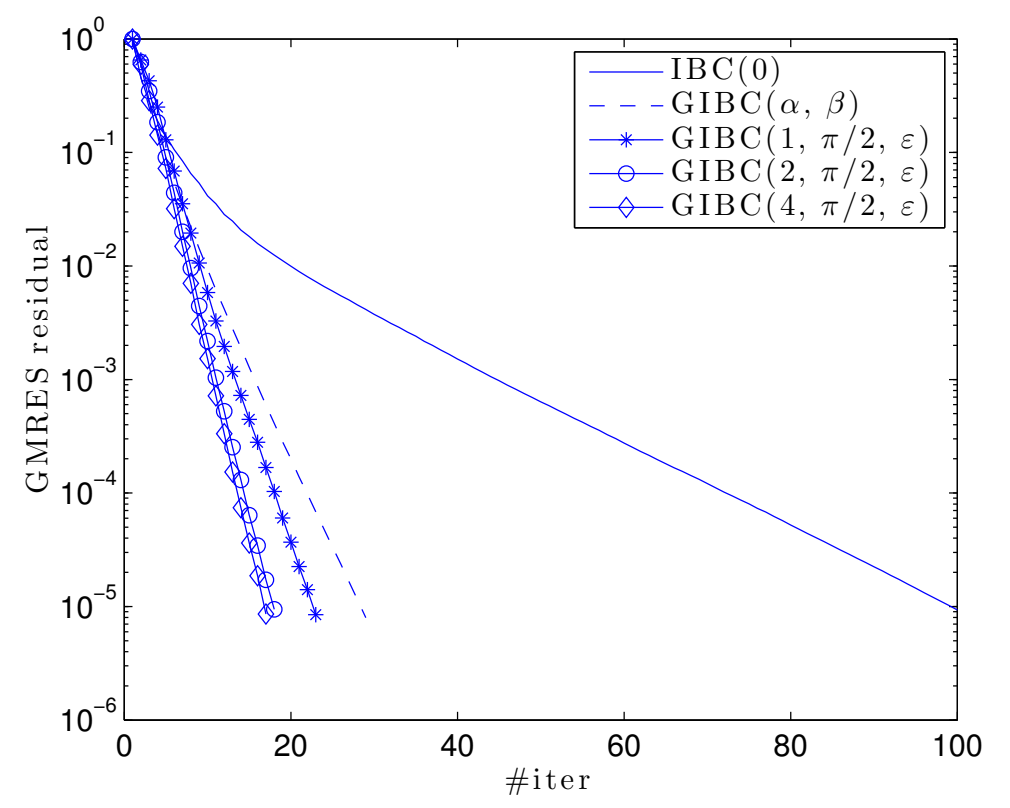

Figure 11: Falcon jet: residual history of the GMRES without restart $\left(N_{\text {dom }}=4, \lambda=10, n_{\lambda}=10\right)$. 